\title{
Wave-induced topographic formstress in baroclinic channel flow
}

\author{
Dirk Olbers*, Karsten Lettmann* and Jörg-Olaf Wolff ${ }^{\dagger}$ \\ *Alfred Wegener Institute for Polar and Marine Research, Bremerhaven, Germany \\ $\dagger$ ICBM, University of Oldenburg, Germany \\ 17th April 2007 revised version
}

\begin{abstract}
Large-scale zonal flow driven across submarine topography establishes standing Rossby waves. In the presence of stratification the wave pattern can be represented by barotropic and baroclinic Rossby waves of mixed planetary-topographic nature which are locked to the topography. In the balance of momentum the wave pattern manifests itself as topographic formstress. This wave-induced formstress has the net effect of braking the flow and reducing the zonal transport. Locally it may lead to acceleration and the parts induced by the barotropic and baroclinic waves may have opposing effects. This flow regime occurs in the circumpolar flow around Antarctica.

The different roles which the wave-induced formstress plays in homogeneous and stratified flows through a zonal channel is analyzed with the BARBI model. It is used in complete form and in a low-order version to clarify the different regimes. It is shown that the barotropic formstress arises by topographic locking due to viscous friction and the baroclinic one due to eddy-induced density advection. For the sinusoidal topography used in this study the transport obeys a law in which friction and wave-induced formstress act as additive resistances, and windstress, the effect of Ekman pumping on the density stratification, and the buoyancy forcing (diapycnal mixing of the stratified water column) of the potential energy stored in the stratification act as additive forcing functions. The dependence of the resistance on the systems parameters (lateral viscosity $\epsilon$, lateral diffusivity $K$ of eddy density advection, Rossby radius $\lambda$, and topography height $\delta$ ) as well as the dependence of transport on the forcing functions are determined. While the current intensity in a channel with homogeneous density decreases from the viscous flat bottom case in an inverse quadratic law $\sim \delta^{-2}$ with increasing topography height and always depends on $\epsilon$, a stratified system runs into a saturated state in which the transport becomes independent of $\delta$ and $\epsilon$ and is determined by the density diffusivity $K$ rather than the viscosity: $K / \lambda^{2}$ acts as a vertical eddy viscosity and the transport is $\lambda^{2} / K$ times the applied forcing. Critical values for the topographic heights in these regimes are identified.
\end{abstract}

\section{Introduction}

Ocean currents are driven by the action of wind and differential buoyancy flux at the surface. Estimating the relative importance of these forces in the generation of the ocean circulation is a delicate matter. Stepping from homogeneous conditions to a stratified ocean model and 
introducing thermohaline stratification in addition to wind forcing not only changes the vertical structure of currents, it also yields fundamentally different transports of the major current systems. This was exemplified in a 'classical' suite of model solutions of the global ocean circulation described in Bryan and Cox (1972) and Cox (1975) obtained with the then state of the art OGCM of the GFDL at Princeton. The most drastic changes in these numerical solutions occur in the flow around Antarctica, the Antarctic Circumpolar Current (ACC). For the geographical conditions of the ACC, models with flat bottom yield a transport which is an order of magnitude too high compared to observations (see e.g. the summary of ACC measurements by Rintoul et al. 2001 or Olbers et al. 2004), while homogeneous (= unstratified) models with realistic submarine topography have a transport which is an order of magnitude too small. The inclusion of stratification, even if only prescribed from initial conditions, then generally gives approximately correct transports. The flat case has an almost zonal circulation, for the homogeneous case it follows mostly the $f / h$-contours, while the stratified case is a broad diffusive current (unless an eddy-resolving model is used), however with the characteristic northward shift leaving Drake Passage and the slow southward motion over the whole Southern Ocean. In the present study we analyze the above described Bryan-Cox scenario in greater detail with an analytical model of a wind- and buoyancy-driven flow through a periodic zonal channel.

The broad range of transport values found in the Bryan-Cox scenario is not known from models of other current systems in the World Ocean with equivalent model ingredients. The reason has been sought in a combination of various features special to the ACC: the zonally unrestricted domain of the path of the ACC, which prevents net pressure gradients in the main body of the fluid to oppose the eastward wind (see e.g. Stommel 1957), the particular pattern of the geostrophic contours which expel the $f / h$-contours from the Drake Passage (see e.g. Cox 1975), the bottom form stress mechanism which acts as the principal sink of momentum (Munk and Palmèn 1951), and the diabatic processes induced by eddies and small scale mixing (for a summary see Olbers et al. 2004). The interplay of these features and the particular role of eddy diffusion in shaping the ACC and its transport was discussed by Olbers et al. (2006) who have reproduced the Bryan-Cox scenario using a simple ocean circulation model with reduced vertical representation and physics (the BARBI model, see below). They showed that the ACC is in a regime which is strongly influenced by high topography but in which steering along $f / h$-contours is overcome by baroclinicity. Likewise, viscous transport of momentum and vorticity is dwarfed by the effect of eddy diffusion of density, finding its manifestation in these balances by residual circulation physics.

The BARBI model is used as well in this study. The BARBI system is briefly discussed in Appendix A (see also Olbers and Eden 2003). It describes the dominant interaction between the barotropic and baroclinic state variables of the full dynamical system of ocean circulation physics by drastic simplification of the oceanic equations of motion. The resulting equations are two-dimensional: the vertically integrated balance of momentum (or the corresponding vorticity balance) and a balance for the potential energy stored in the stratification. Besides the complete form of BARBI we also use a low-order version in which the degrees of freedom are further greatly reduced. The latter approach should help to interpret the full model results and entangle the physics of the Bryan-Cox scenario. The low-order version truncates the model variables to a small set of zonal modes which represent a system of coupled barotropic-baroclinic Rossby waves with consideration of topography, viscous and diffusive diabatic effects, and nonlinearity due to density advection.

The new concept proposed in this study is wave-induced topographic resistance. The 
crucial variable is the bottom formstress induced by large-scale Rossby waves which excite characteristic bottom pressure variations in response to the flow crossing the topography. We reveal and discuss in detail the mechanisms which set the wave-induced topographic formstress which generally opposes the zonal acceleration of the fluid and decreases the ACC transport. We derive resistance functions, relating the transport to the applied forcing, for the above discussed regimes, admittedly for a simple sinusoidal topography.

The paper is organized as follows. Section 2 discusses numerical experiments with a full OGCM and with BARBI for wind and buoyancy-driven flow through a periodic zonal channel. The equations governing a baroclinic, topographically influenced flow through a reentrant channel are outlined in section 3 and treated in section 4 by a low-order approach for the zonal dependence. We discuss the solution of the linearized model in section 5 and introduce the concept of topographic wave resistance to describe the behavior of the linear barotropic and baroclinic formstress components in the zonal balance of momentum. The theory is extended in section 6 to include advection of density which leads to a supercritical regime of the ACC where the current speed exceeds the speed of baroclinic waves. The theory of wave-induced resistance thus becomes nonlinear. The last section is a concluding discussion of our findings. We have added three appendices. In Appendix A we give a short summary of the BARBI model. In Appendix B we discuss the flat-bottom and topographic wave properties of the low-order model, and Appendix $C$ clarifies the energy conservation of the BARBI low-order model.

\section{The Bryan-Cox scenario for a zonal channel flow}

To elucidate the circulation regimes analyzed in the present study we have simulated the channel flow with a full three-dimensional OGCM (the Hamburg-Ocean-Primitive-Equation model HOPE, Wolff et al. 1997) and with the BARBI model for the sinusoidal topography used in the analytical model with four heights, both for barotropic and for baroclinic conditions (see Figure 1). The model parameters are those of the standard conditions given below in Table 1. The BARBI simulation is driven by the windstress and the buoyancy forcing specified below in equation (7). The forcing in the HOPE model is by the same wind but has a temperature restoring at the surface increasing from $0^{\circ} \mathrm{C}$ at the southern boundary to $10^{\circ} \mathrm{C}$ at the northern boundary, with a meridional dependence $3 y / 8-(1 / 4) \cos y \sin y\left(\sin ^{2} y+3 / 2\right)$ (this structure solves the flat bottom diffusion problem with the buoyancy forcing specified for the BARBI runs). The HOPE model uses a Cartesian grid with a grid distance of $100 \mathrm{~km}$ and 13 vertical levels, the domain has a length $4000 \mathrm{~km}$ and a nominal width $1800 \mathrm{~km}$ and is located with its central latitude at $60^{\circ} \mathrm{S}$. The BARBI simulation uses a spherical grid of $72^{\circ}$ in longitude between $-66^{\circ}$ and $-52^{\circ}$ in latitude with a resolution of $1^{\circ} \times 1^{\circ}$ and two Rossby wave modes as described later in the text. Both models have been integrated for 20 years and the baroclinic cases are not yet entirely stationary. The simulations of HOPE and BARBI are very similar but not identical. Both do not exactly reproduce the theoretical transport for a flat bottom, which is $2264 \mathrm{~Sv}$. This is because of the cubic dependence of transport on the channel width which is not well defined on the model grids. The overall dependence of the circulation pattern and the total transport on the topography height are, however, well simulated. The experiments capture the basic features of the Bryan-Cox scenario described above. We also refer to a corresponding eddy-resolving quasigeostrophic (QG) simulation driven by a similar windstress over similar sinusoidal topography with a height of $500 \mathrm{~m}$ 


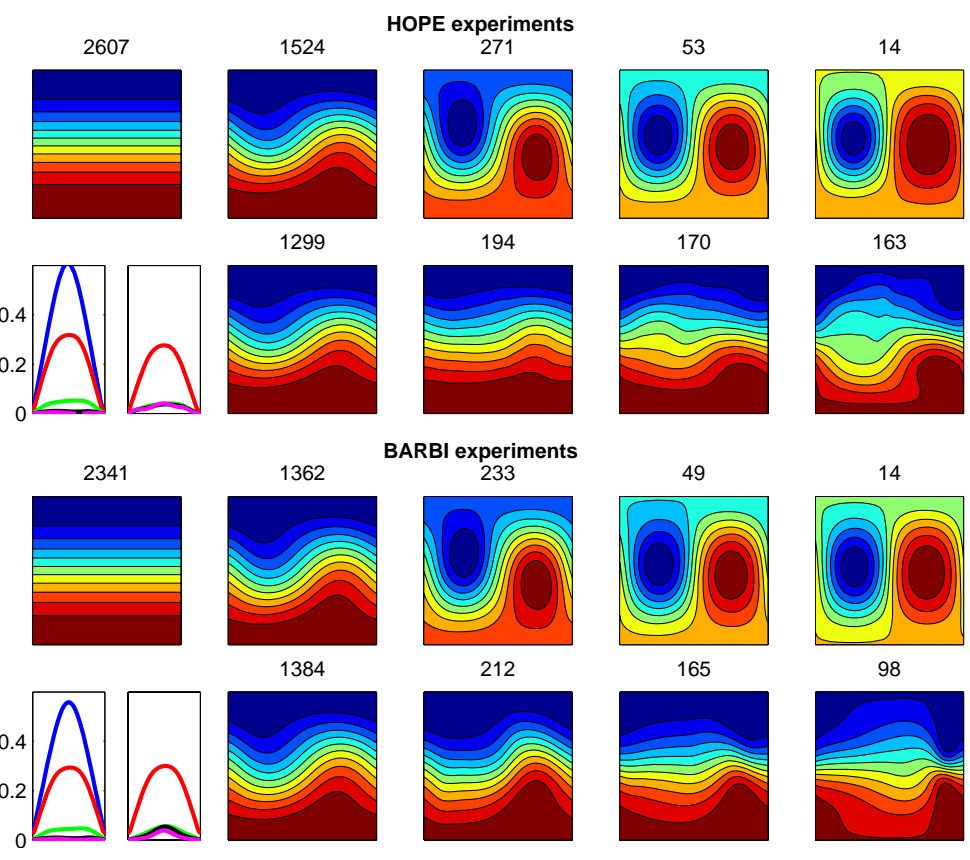

Figure 1: Upper panels: streamfunction of barotropic [first row] and baroclinic [second row] HOPE experiments for different topography heights $(0,100,400,1000$ and $2000 \mathrm{~m}$ from left to right) after 20 years of integration. The topography is sinusoidal in both directions, as shown in the second case of Figure 2. In the left panels of the second row the profiles of zonal mean velocity $\left(\mathrm{in} \mathrm{ms}^{-1}\right.$ ) are shown (left subpanel: barotropic, right subpanel: baroclinic experiments; colors from blue to magenta refer to increasing topography height). Lower panels: same for the corresponding BARBI experiments. The transport (in Sv) is given in the title of each panel. There are 10 equidistant contour lines between minimum and maximum in each plot.

(see figures 10 and 11 in Olbers and Völker 1996 and figure 4 in Völker 1999). The time mean QG circulation show similarity to the coarse resolution simulations with HOPE or BARBI but it is not clear to which real topography height the QG height would correspond. There are numerous other coarse or eddy resolving simulations of a periodic zonal flow in a channel or in a box ocean with a southern passage, e.g. Gnanadesikan and Hallberg (2000), MacCready and Rhines (2001) and Tansley and Marshall (2001). A notable collection of eddy resolving QG channel simulations is found in Walsteijn (1996), and Borowski (2003) discusses a huge number of sensitivity studies with coarse resolution channel models. In these studies the relation between the transport and the wind strength and its meridional shape is in foreground, not the dependence on the height of topography which is in the center of the present study and the Bryan-Cox scenario.

\section{BARBI equations for channel flow}

A zonal channel is considered in the coordinate domain $0 \leq x \leq 2 \pi$ and $0 \leq y \leq \pi$. The topography height $h$ is taken as sinusoidal in the zonal direction $x$, and the Coriolis parameter $f$ will be considered for a $\beta$-plane (in the southern hemisphere) 


$$
h(x, y)=1+\eta(y) \sin x \quad \text { and } \quad f(y)=-1+\beta\left(y-\frac{\pi}{2}\right)
$$

where $\eta(y)=\delta \zeta(y)$ is the meridional shape of the topography with amplitude $\delta$, and $\beta$ the scaled planetary vorticity gradient ${ }^{1}$. We assume that the topography vanishes on the walls, i.e. $\zeta=0$ at $y=0, \pi$.

We base our model on the BARBI physics (Olbers and Eden 2003) and consider dimensionless variables and equations (more details are given in Appendix A). The fundamental variables describing the flow are the vertically integrated velocity $\mathbf{U}=\int \mathbf{u} d z=$ $(-\partial \psi / \partial y, b \partial \psi / \partial x)$ or its streamfunction $\psi$, and the baroclinic potential energy $h^{2} \phi=g \int z \rho d z$ stored in the density profile $\rho$ (integration is from top to the ocean depth $z=-h$ ). The scaled vertically integrated balances of zonal and meridional momentum are ${ }^{2}$

$$
\begin{aligned}
-\left[\frac{\partial}{\partial t}-\epsilon \nabla^{2}\right] \frac{\partial \psi}{\partial y}-f \frac{\partial \psi}{\partial x} & =-h \frac{\partial P}{\partial x}-\frac{\partial}{\partial x} h^{2} \phi+\tau \\
b^{2}\left[\frac{\partial}{\partial t}-\epsilon \nabla^{2}\right] \frac{\partial \psi}{\partial x}-f \frac{\partial \psi}{\partial y} & =-h \frac{\partial P}{\partial y}-\frac{\partial}{\partial y} h^{2} \phi
\end{aligned}
$$

with the diffusion coefficient $\epsilon$ (lateral Ekman number); $b$ is the ratio of the horizontal channel dimensions. The scaled balance of the baroclinic potential energy is

$$
\begin{array}{r}
b\left[\frac{\partial}{\partial t}-\kappa \nabla^{2}\right] h^{2} \phi+\gamma h \mathcal{J}(\psi, \phi)+\frac{1}{2} \lambda^{2}\left(\mathcal{J}\left(h^{2} \phi, \frac{h^{2}}{f}\right)-\mathcal{J}(\psi, h)\right)= \\
=b\left(\mathcal{Q}-\mathcal{D}[\phi]-\frac{1}{2} \lambda^{2} \operatorname{curl} \frac{h^{2} \boldsymbol{\tau}}{f}\right)
\end{array}
$$

Further dimensionless control parameters appear: $\lambda$ is a measure of the scaled baroclinic Rossby radius; $\gamma$ measures the nonlinearity due to density advection; and $\kappa$ is a scaled GentMcWilliams diffusivity for density (see Olbers et al. 2006 for a derivation). We will consider (4) in full form and in linearized form as well, obtained by putting $\gamma=0$. In view of the smallness of this parameter in comparison to the others in (4), linearization seems to be justified but it should be borne in mind that the scaled fields are not of order unity. With the enormous range of variations which can be achieved in the system by changing the topographic height $\delta$ (see e.g. Figure 4 below) such a scaling is impossible. An intermediate scaling has been chosen, basically by scaling the streamfunction and the potential energy using a windstress amplitude, the channel width and depth and the Coriolis parameter (see Appendix A).

${ }^{1}$ All scaling expressions and standard values of the system parameters are listed below in Table 1 and Appendix A.

${ }^{2}$ We use the abbreviations

$$
\boldsymbol{\nabla}=\left(b \frac{\partial}{\partial x}, \frac{\partial}{\partial y}\right) \quad \nabla^{2}=b^{2} \frac{\partial^{2} \psi}{\partial x^{2}}+\frac{\partial^{2} \psi}{\partial y^{2}} \quad \mathcal{J}(A, B)=b\left[\frac{\partial A}{\partial x} \frac{\partial B}{\partial y}-\frac{\partial A}{\partial y} \frac{\partial B}{\partial x}\right]
$$

for the scaled gradient, Laplacian and Jacobian operators. 


$$
\begin{array}{l|c|c}
\epsilon=\frac{A_{h} \pi^{2}}{\left|f_{0}\right| B^{2} b}=2.7 \times 10^{-4} & \kappa=\frac{K \pi^{2}}{2 B^{2}\left|f_{0}\right| b}=1.3 \times 10^{-5} & b=\frac{2 B}{L}=0.9 \\
\hline \gamma=\frac{\pi T_{0}}{B h_{0} f_{0}^{2}}=2.7 \times 10^{-6} & \lambda^{2}=\frac{\pi^{2}}{3}\left(\frac{N h_{0}}{f_{0} B}\right)^{2}=6.9 \times 10^{-3} & \beta=\frac{B}{\pi a}=5.2 \times 10^{-2}
\end{array}
$$

Table 1: Standard values of the dimensionless parameters of the model. The dimensional parameters are: $L=$ $4000 \mathrm{~km}$ and $B=1800 \mathrm{~km}$ are length and width of the channel, $A_{h}=10^{4} \mathrm{~m}^{2} \mathrm{~s}^{-1}$ and $K=10^{3} \mathrm{~m}^{2} \mathrm{~s}^{-1}$ are horizontal viscosity and eddy diffusivity, $h_{0}=4000 \mathrm{~m}$ is the mean depth, $f_{0}=-1.26 \times 10^{-4} \mathrm{~s}^{-1}$ is the Coriolis parameter at center latitude of the channel, $a=11035 \mathrm{~km}$ is the earth radius times the tangens of the center latitude $60^{\circ} \mathrm{S}, N=2.6 \times 10^{-3} \mathrm{~s}^{-1}$ is the Brunt-Väisälä frequency, and $T_{0}=$ $10^{-4} \mathrm{~m}^{2} \mathrm{~s}^{-2}$ a windstress scale. The (dimensioned) internal Rossby radius is $N h_{0} /\left(\left|f_{0}\right| \pi\right)=26 \mathrm{~km}$, the scaled speed of baroclinic Rossby waves is $c=\beta \lambda^{2} / 2=1.8 \times 10^{-4}$.

We will refer to (3) and (4) as the barotropic and baroclinic equations, respectively, and call $\psi$ barotropic and $\phi$ baroclinic variables. It should be emphasized that the presence of topography couples $\psi$ and $\phi$, describing then a mixed stratified state. Consequently, we call a state with nonzero $\psi$ and $\phi$ a coupled or mixed state. A state with $\phi \equiv 0$ is a barotropic state and with $\psi \equiv 0$ a baroclinic state. For later reference we present the vorticity equation of the system,

$$
\frac{\partial}{\partial t} \boldsymbol{\nabla} \cdot \frac{1}{h} \boldsymbol{\nabla} \psi+\mathcal{J}\left(\psi, \frac{f}{h}\right)=-\frac{\partial}{\partial y}\left(\frac{\tau}{h}\right)+\mathcal{J}(h, \phi)+\epsilon \boldsymbol{\nabla} \cdot \frac{1}{h} \nabla^{2} \boldsymbol{\nabla} \psi
$$

built from (3) by elimination of the bottom pressure $P$. In addition to the wind curl, vorticity is forced by a torque-like term $\mathcal{J}(h, \phi)$, the JEBAR term of Sarkisyan and Ivanov (1971). Equations (4) and (5) bear some similarity to a two-layer QG model. Indeed, the vorticity equation corresponds to the QG balance of the depth integrated transport if the nonlinear advection terms are abandoned there. Furthermore, if the QG balance of the baroclinic streamfunction (upper minus lower layer streamfunctions) is considered for scales exceeding the baroclinic Rossby radius we find a certain similarity to the above balance of potential energy, with $h^{2} \phi$ as baroclinic streamfunction. There are differences because BARBI is valid for arbitrary topography while QG needs infinitesimal slopes. Also, BARBI still contains the geostrophic term while the QG vorticity equation is formulated as an ageostrophic balance.

The system is forced by the windstress $\tau$ and the source $\mathcal{Q}$ of potential energy. The derivation of the BARBI equations by Olbers and Eden (2003) shows that $\mathcal{Q}$ is the vertical integral of the vertical turbulent buoyancy flux. Turbulent mixing apparently changes the potential energy. We assume a zonal wind stress which, moreover, is zonally constant, i.e. $\boldsymbol{\tau}=(\tau(y), 0)$. The integrated buoyancy flux is a given source of potential energy, it is also taken zonally constant, thus $\mathcal{Q}=\mathcal{Q}(y)$. We thus exclude externally forced zonal gradients of the baroclinic potential energy field.

In addition to the topography profile $\eta(y)$ there are two forcing profiles $\tau(y)$ and $\mathcal{Q}(y)$ free to choose, and there are the seven above mentioned system parameters $\delta, \epsilon, \kappa, \lambda, b, \beta$, and $\gamma$. Note that for steady states the parameters $\kappa, \lambda$ and $\gamma$ appear only as $\kappa / \lambda^{2}$ and $\gamma / \lambda^{2}$. A huge number of substantially differing solutions (or solution regimes) can be generated by varying the profiles and parameters, and thus, we must obviously confine the analysis 


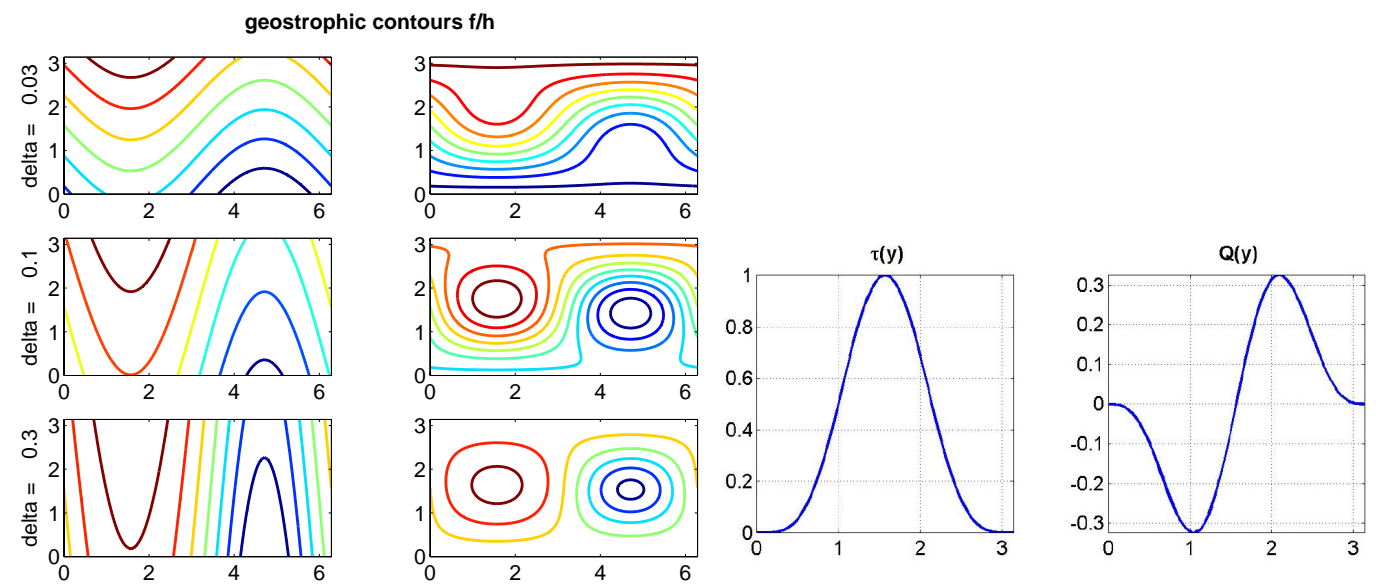

Figure 2: Left: $f / h$-contours for the sinusoidal topography (1) with $\zeta=1$ (left subpanels) $\zeta(y)=\sin ^{2} y$ (right subpanels) for increasing values of the topography height $\delta$ (the examples in the panels have $\delta=0.03,0.1$ and 0.3$)$. Right: the profiles of the forcing functions for windstress $\tau(y)$ and potential energy source $\mathcal{Q}(y)$, given by (7) (with $\tau_{0}=1, Q_{0}=-\tau_{0} \lambda^{2} / 2$ ).

to a set of basic experiments. Consequently we study the channel flow for fixed system parameters $\epsilon, \kappa, \lambda, b, \beta$ in a linearized version $(\gamma=0)$ and in a nonlinear version $(\gamma \neq 0)$, taking the standard values given in Table 1 , and vary only the topography height $\delta$. Further, we consider the 'unblocked' topography

$$
\eta(y)=\delta \sin ^{2} y
$$

(see right panel of Figure 2 and remarks in the next section) and take the forcing profiles (see Figure 2)

$$
\tau(y)=\tau_{0} \sin ^{4} y \quad \mathcal{Q}(y)=Q_{0} \cos y \sin ^{3} y
$$

The wind is concentrated in the channel center and vanishes on both boundaries (it has net zero curl). The potential energy source $\mathcal{Q}$ describes a loss (for $Q_{0}<0$ ) of potential energy in the southern half and a corresponding gain in the northern half of the channel (it integrates to zero over the channel). Note that $\partial \tau / \partial y$ mirrors the shape function of $\mathcal{Q}(y)$.

To have a sink for zonal momentum we take no-slip conditions, $\psi^{\prime}=0$, at the southern and northern boundaries ${ }^{3}$. Mass conservation requires $\psi=0$ at $y=0$ and $\psi=-\Psi_{n}$ at $y=$ $\pi$. The total transport through the channel $\Psi_{n}$ is part of the solution. Boundary conditions for the potential energy balance must be considered with care. For the linear system and a net zero $\mathcal{Q}$-forcing we take zero flux $\left(h^{2} \phi\right)^{\prime}=0$ at both boundaries. With a nonlinear potential energy equation there is generally a net transfer between the kinetic energy and the potential energy and hence, a net flux $\Phi_{n}$ across the boundaries or interior dissipation must balance this net interior source/sink. We will assume zero flux at the southern boundary, $\left(h^{2} \phi\right)^{\prime}=0$, and a nonzero flux $\kappa\left(h^{2} \phi\right)^{\prime}=\Phi_{n}$ at the northern boundary which is open in the real Southern

\footnotetext{
${ }^{3}$ The prime denotes the derivative with respect to $y$.
} 
Ocean. Notice that $\Phi_{n}$ must as well be determined by the solution. Alternatively (or in addition) a dissipation term $\mathcal{D}[\phi]$ can be implemented (see e.g. Olbers et al. 2006). In this study the dissipation term is set to zero.

\section{A truncated wave model}

The model equations (3) and (4) will be extremely simplified to gain a set which is tractable by analytical means but still contains the basic physical processes which determine the transport through the channel as response to the applied forcing. The response in the resulting fields $\psi$ and $\phi$ is assumed to be confined to the zonal wavenumber of the topography. We thus search a truncated solution of the form

$$
\psi(x, y)=\psi_{0}(y)+\psi_{S}(y) \sin x+\psi_{C}(y) \cos x
$$

and likewise for $P$ and $\phi$. Note that $U_{0}=-\psi_{0}^{\prime}$ is the zonal mean transport velocity.

We should mention that the above representation is not well suited in special configurations, namely in unstratified flows with blocked $f / h$-contours. In fact, analytical and numerical solutions (Krupitsky and Cane 1994, Wang and Huang 1995) of barotropic channel flow reveal a current regime in which the flow is in narrow frictional boundary layer currents at the walls, switching sides from south to north in a narrow internal layer along a connecting $f / h$-contour. This situation is entirely unrealistic for the ACC and does not occur if stratification is included (see the analysis of Olbers et al. 2006 and the results in this study). Blocking of $f / h$-contours does not occur if the topography vanishes at both walls, i.e. $\eta(0)=\eta(\pi)=0$. The blocked and unblocked cases are exemplified in Figure 2 showing the topography and the geostrophic contours $f / h$ for various heights $\delta$ and the meridional structure function $\zeta(y)=1$ and $\zeta(y)=\sin ^{2} y$, respectively.

We refer to $U_{0}$ and $\phi_{0}$ as zonal components and to $\psi_{S, C}$ and $\phi_{S, C}$ as barotropic and baroclinic nonzonal or wave components, respectively. Remember, however, that due to the topographic coupling the $\psi$ and $\phi$ variables are neither strictly barotropic nor baroclinic (the eigenvectors are of mixed quality).

In usual low-order models not only the $x$-dependence but also the $y$-dependence of the system is represented by a small set of structure functions (see e.g. Olbers and Völker 1996, Völker 1999, Borowski 2003, Olbers et al. 2004, and the more fundamental work of Charney and DeVore 1979). We refrain from such further truncation of the spatial dependence of the model equations because it is found by direct integration (see next sections) that the $y$ profiles of the system components have a rather rich structure and, moreover, the following analysis can mostly be done without further simplifications.

\subsection{Barotropic equations}

Projecting the momentum balance onto $1, \sin x$ and $\cos x$ yields 


$$
\begin{aligned}
\left(\frac{\partial}{\partial t}-\epsilon \frac{\partial^{2}}{\partial y^{2}}\right) U_{0} & =\frac{1}{2} \eta P_{C}+\tau \\
-\left(\frac{\partial}{\partial t}-\epsilon \mathcal{L}\right) \psi_{S}^{\prime}+f \psi_{C} & =P_{C}+\phi_{C} \\
\left(\frac{\partial}{\partial t}-\epsilon \mathcal{L}\right) \psi_{C}^{\prime}+f \psi_{S} & =P_{S}+\left(\phi_{S}+2 \eta \phi_{0}\right) \\
f U_{0} & =-P_{0}^{\prime}-\frac{1}{2} \eta P_{S}^{\prime}-\left(\phi_{0}+\eta \phi_{S}\right)^{\prime} \\
b^{2}\left(\frac{\partial}{\partial t}-\epsilon \mathcal{L}\right) \psi_{C}+f \psi_{S}^{\prime} & =P_{S}^{\prime}+\eta P_{0}^{\prime}+\left(\phi_{S}+2 \eta \phi_{0}\right)^{\prime} \\
b^{2}\left(\frac{\partial}{\partial t}-\epsilon \mathcal{L}\right) \psi_{S}-f \psi_{C}^{\prime} & =-P_{C}^{\prime}-\phi_{C}^{\prime}
\end{aligned}
$$

Here,

$$
\mathcal{L}=\partial^{2} / \partial y^{2}-b^{2}
$$

derives from the Laplacian operator. Equation (12) states that the zonal mean flow $U_{0}=-\psi_{0}^{\prime}$ is geostrophic with respect to the combination of the gradients of the bottom pressure and the potential energy. The velocities of nonzonal components are affected by inertia and friction. Equation (9) is the dynamical balance of the zonal momentum: the wind stress is balanced by inertia, friction and the bottom formstress $\eta P_{C} / 2$.

Simplified barotropic equations The set of equations (9) to (14) can be simplified further. We form vorticity equations from the above barotropic wave equations and determine $P_{S, C}$ from Poisson equations which are derived by applying appropriate divergences. To very high accuracy the solutions of the latter may be approximated by

$$
P_{C} \approx f \psi_{C}-\phi_{C} \quad P_{S} \approx f \psi_{S}-\left(\phi_{S}+2 \eta \phi_{0}\right)
$$

This was checked by numerical integration. We shall refer to the individual parts $\eta f \psi_{C} / 2$ and $-\eta \phi_{C} / 2$ of the formstress $\eta P_{C} / 2$ as barotropic and baroclinic bottom formstress components, respectively. The term $\eta P_{S}^{\prime} / 2$ in (12) makes the profile of $U_{0}$ slightly asymmetric (see Figure 3 below) but has no significant influence on the channel transport. Also this was checked by numerical integrations. For some analytical arguments presented below we shall ignore this term and arrive at an reduced model for the barotropic system

$$
\begin{aligned}
\left(\frac{\partial}{\partial t}-\epsilon \frac{\partial^{2}}{\partial y^{2}}\right) U_{0} & =\frac{1}{2} \eta\left(f \psi_{C}-\phi_{C}\right)+\tau \\
\left(\frac{\partial}{\partial t}-\epsilon \mathcal{L}\right) \mathcal{L} \psi_{C}+\beta \psi_{S} & =\eta\left[f U_{0}+\left(\phi_{0}+\eta \phi_{S}\right)^{\prime}\right] \\
\left(\frac{\partial}{\partial t}-\epsilon \mathcal{L}\right) \mathcal{L} \psi_{S}-\beta \psi_{C} & =0
\end{aligned}
$$


In (17) the bottom formstress appears now as $F=\eta\left(f \psi_{C}-\phi_{C}\right) / 2$ and in (18) we see the JEBAR torque in the form $\eta\left(\phi_{0}+\eta \phi_{S}\right)^{\prime}$. The term $\eta f U_{0}$ of the rhs and the $\beta$-term on the lhs of (18) derive form the planetary-topographic Jacobian (the barotropic stretching term) in the vorticity balance. Notice that coupling to the baroclinic system is inactive if the bottom is at a constant depth.

\subsection{Baroclinic equations}

In a similar way the truncated expansion (8) and projection of the balance (4) of the potential energy $h^{2} \phi$ yields the baroclinic low-order equations. They are fairly complicated if all terms are retained. We proceed with a slightly simplified set of equations in which terms of order $\beta \lambda^{2}, \cdots, \lambda^{2} \delta^{3}$ are omitted,

$$
\begin{aligned}
\left(\frac{\partial}{\partial t}-\kappa \frac{\partial^{2}}{\partial y^{2}}\right)\left(\phi_{0}+\eta \phi_{S}\right) & =\mathcal{Q}+\frac{1}{2} \lambda^{2}\left[-\eta \psi_{C}+\eta \phi_{C} / f+\tau / f\right]^{\prime}+\frac{1}{2} \gamma \mathcal{A}_{0} \\
\left(\frac{\partial}{\partial t}-\kappa \mathcal{L}\right) \phi_{C}-c\left(\phi_{S}+2 \eta \phi_{0}\right) & =\lambda^{2} \eta\left[U_{0}+\left(\phi_{0}+\eta \phi_{S}\right)^{\prime} / f\right]+\frac{1}{2} \gamma \mathcal{A}_{C} \\
\left(\frac{\partial}{\partial t}-\kappa \mathcal{L}\right)\left(\phi_{S}+2 \eta \phi_{0}\right)+c \phi_{C} & =\frac{1}{2} \gamma \mathcal{A}_{S}
\end{aligned}
$$

Here $c=\beta \lambda^{2} /\left(2 f^{2}\right)$ is the speed of baroclinic (flat bottom) Rossby waves. The equations are easy to interpret: the zonal mean potential energy $\phi_{0}+\eta \phi_{S}$ can directly be forced by either the external source $\mathcal{Q}$ or by the Ekman pumping $(\tau / f)^{\prime}$ acting on the background stratification. Notice that the $\mathcal{Q}$-forcing can only become significant if the amplitude is of order $\lambda^{2}(\tau / f)^{\prime}$ or larger. The terms proportional to $\lambda^{2} \eta$ in the above balances describe linear topographic baroclinic wave processes, due to lifting or lowering the background stratification when the current crosses the topography. The $\mathcal{A}$-terms derive from advection. They are quadratic in the field variables and will be discussed later in section 6 . We continue with the steady state form of the above equations.

Balance of shear and layer momentum It is worth considering a reformulation of the zonal baroclinic balance (20). The gradient of zonal mean potential energy may be used to define the transport variable $S_{0}=-\left(\phi_{0}+\eta \phi_{S}\right)^{\prime} / f$. In fact, from (12) we note that while $U_{0}$ is the total transport, the variable $S_{0}$ is the geostrophic baroclinic transport, and $U_{0}-S_{0}$ is the transport with reference to the bottom. The balance (20) can be interpreted as balance of a baroclinic (shear) momentum. For steady state conditions,

$$
\begin{aligned}
0 & =-\frac{\kappa}{\lambda^{2}} S_{0}-\frac{1}{2} \eta\left(f \psi_{C}-\phi_{C}\right)+\mathcal{N}_{0}+\mathcal{S} \\
\mathcal{S} & =\frac{1}{2} \tau+\frac{1}{f \lambda^{2}} \int_{0}^{y} \mathcal{Q} d y^{\prime}
\end{aligned}
$$

Notice the similarity to the barotropic momentum balance (17): the diabatic momentum flux (first term on the rhs) is balanced by formstress, the baroclinic forcing $\mathcal{S}$ (arising from Ekman pumping and the $\mathcal{Q}$-source) and a nonlinear term $\mathcal{N}_{0}$ which derives from $\mathcal{A}_{0}$. The diabatic 
term is the transient eddy interfacial formstress (of Gent-McWilliams form). It is supplemented by the bottom formstress $\eta\left(f \psi_{C}-\phi_{C}\right) / 2$, the interfacial formstress due to standing eddies (the nonlinear term $\mathcal{N}_{0}$ ) to transport momentum vertically. Both interfacial terms are absent in the barotropic balance. The standing interfacial formstress will be analyzed later in section 6. Adding the balances for the total and the shear momentum we arrive at the balance of upper layer momentum,

$$
0=\epsilon U_{0}^{\prime \prime}-\frac{\kappa}{\lambda^{2}} S_{0}+\mathcal{N}_{0}+\tau+\mathcal{S}
$$

in which frictional and interfacial stresses are active but the bottom formstress is absent. Similarly, a balance of momentum for the bottom layer can be built.

\section{Linear theory}

The coupled barotropic and baroclinic equations (9) to (14) (or in slightly simplified form (17) to (19)) and (20) to (22) constitute the mixed planetary-topographic wave problem, with forcing by windstress and buoyancy flux as well as friction and diffusion included. For flat bottom and non-steady conditions, the familiar barotropic and baroclinic planetary Rossby waves are recovered. The presence of topography modifies and couples the wave equations to describe mixed barotropic-baroclinic planetary-topographic Rossby waves. This is shown in Appendix B (for a general treatment of topographic waves see e.g. Rhines 1977).

Flat bottom Even in the highly simplified form it is hopeless to search for a general analytical solution for the above equations. A trivial exception, of course, is the case of flat topography, $\eta \equiv 0$, where the flow is zonal. In steady state $\epsilon U_{0}^{\prime \prime}+\tau=0$ and $\psi_{S, C} \equiv 0$. For later reference we mention the solution for the zonal transport driven by the windstress (7),

$$
\int_{0}^{\pi} U_{0} d y=\frac{\tau_{0}}{128 \epsilon} \pi\left(15+4 \pi^{2}\right)
$$

For the standard parameters the total transport is 4990 in dimensionless units ( $\equiv 2264 \mathrm{~Sv}$ ). Because we have excluded an external non-zonal forcing the baroclinic nonzonal parts $\phi_{S, C}$ vanish as well and the baroclinic problem reduces to the linear diffusive equation for the zonal mean potential energy field, $\kappa \phi_{0}^{\prime \prime}+\mathcal{Q}+\lambda^{2}(\tau / f)^{\prime} / 2=0$.

Barotropic state The behavior of the transport in a barotropic system can roughly be described from the simplified equations (17) to (19). The vorticity balances approximately yield $\psi_{C} \sim \epsilon \eta f U_{0} /\left(\epsilon^{2}+\beta^{2}\right)$ and then, from (17), we find

$$
U_{0} \sim \frac{\tau / \epsilon}{1+\frac{1}{2} \eta^{2} / \eta_{\text {trop }}^{2}}
$$

where $\eta_{\text {trop }} \sim \beta$. More precise expressions will be derived below. Here we only want to point out the (unlimited) quadratic decrease of transport by increasing topography height. It is obvious from (26) (and trivial) that a steady barotropic state cannot exist without friction. This contrasts the unblocked topography the blocked one: according to Krupitzky and 
Cane (1994) a blocked topography in a barotropic case yields a transport which becomes independent of friction at large heights.

The state (26) is achieved as a stationary response of the barotropic planetary-topography Rossby wave $\left(\psi_{C}, \psi_{S}\right)$ to the forcing by the windstress and arrested by friction with a nonzero phase shift relative to the topography. This phase shift is proportional to the friction coefficient (the cosine wave component is the part which is out of phase with respect to the topography). The in-phase component $\psi_{S} \sim \beta \eta f U_{0} /\left(\epsilon^{2}+\beta^{2}\right)$ is non-zero in the limit of zero friction. Note that $\psi_{C} / \psi_{S}=O(\epsilon / \beta) \ll 1$. The above relations between the zonal and the wave components, assuming small $\epsilon$, imply that the flow is along $f / h$ contours. Take $\psi=G(f / h) \simeq G(f)-G^{\prime}(f) f \eta \sin x+\cdots$. This yields $U_{0}=-\beta G^{\prime}(f)$ for the zonal mean current and $\psi_{C}=0, \psi_{S}=-\frac{1}{2} f \eta G^{\prime}(f)=\frac{1}{2}(f / \beta) \eta U_{0}$ which mirrors exactly the above presented relations for $\beta \gg \epsilon \rightarrow 0$.

The purely baroclinic state can be analyzed very similarly and yields a dependence of $S_{0}$ on $\eta$ and $\kappa$ corresponding to (26). The baroclinic formstress is generated by a stationary response of a baroclinic Rossby wave in a similar way but there $\kappa$ generates the phase shift.

Topographic saturation in the coupled system With increasing topography height the $\eta$ terms in the equations become dominant. In the vorticity balance the topographic-planetary Jacobian and the JEBAR must tend to balance and likewise the $\eta$-terms in the baroclinic balance (20) or (23). The latter terms derive from the pumping by barotropic and baroclinic vertical velocities acting on the background stratification (the term proportional to $\lambda^{2}$ in (4)). This state has been described in detail by Olbers et al. (2006), using a BARBI model of a realistic ACC circulation with real topography and forcing. As in that analysis we also get here the 'baroclinic Stommel equation' regime in which density diffusion dominates and $f / h$-characteristics are swept off by the baroclinicity. We arrive at these results either by expanding in terms of $\eta$ or - more conveniently - in terms of $\kappa$ and $c$ (note that these parameters are the smallest in the system, see Table 1). Formally, we assume $\mathcal{Q}$ and $\tau / f$ to be first order.

To zero order we get from (20) and (21)

$$
\begin{aligned}
F^{(0)}=\frac{1}{2} \eta\left(f \psi_{C}^{(0)}-\phi_{C}^{(0)}\right) & =0 \\
J^{(0)}=f U_{0}^{(0)}+\left(\phi_{0}^{(0)}+\eta \phi_{S}^{(0)}\right)^{\prime}=f\left(U_{0}^{(0)}-S_{0}^{(0)}\right) & =0
\end{aligned}
$$

where both terms on the rhs of (18), deriving from the Jacobians in (5), have been abbreviated by $\eta J$. The first relation gives the balance of the barotropic and the baroclinic bottom formstress components to lowest order. The second is the above mentioned balance between the topographic-planetary Jacobian and JEBAR but at the same time it is a geostrophic balance of the zonal current and the pressure gradient imposed by the baroclinic potential energy ( $U_{0} \approx S_{0}$ derives from the geostrophic relation $f u_{z}=g \rho_{y}$, written in terms of transport). The effect of the bottom pressure in the geostrophic balance of the zonal current is thus small. This latter relation is derived in Borowski et al. (2002) by other means and discussed in Olbers et al. (2004). The next order of (20) yields

$$
F^{(1)}=\frac{1}{2} \eta\left(f \psi_{C}^{(1)}-\phi_{C}^{(1)}\right)=-\left(\kappa / \lambda^{2}\right) S_{0}^{(0)}+\frac{1}{2} \tau+1 /\left(f \lambda^{2}\right) \int_{0}^{y} \mathcal{Q} d y^{\prime}
$$


and hence, with $S_{0}^{(0)} \approx U_{0}^{(0)}$ from (28) we obtain

$$
0=\epsilon U_{0}^{\prime \prime}-\frac{\kappa}{\lambda^{2}} U_{0}+\tau+\mathcal{S}
$$

for the zonal balance correct to first order. In this saturated form (30) it is sufficient to determine the zonal flow. It is the zonal mean of the 'baroclinic Stommel equation' derived in Olbers et al. (2006), and here in the low-order form. It is named so because the viscous term may be safely neglected $\left(\epsilon \ll \kappa / \lambda^{2}\right)$ and then, the transport is determined by a second order derivative of the streamfunction,

$$
\frac{\kappa}{\lambda^{2}} \psi_{0}^{\prime \prime}=\frac{3}{2} \tau^{\prime}+\frac{\mathcal{Q}}{f \lambda^{2}}
$$

in the same way as the bottom friction term acts in the flat bottom Stommel equation. The $\beta$-term of Stommel's equation is here absent because of zonal averaging. In the present regime, however, the 'bottom friction' derives from lateral density diffusion, and the coefficient $\kappa / \lambda^{2} \sim K f^{2} /\left(N^{2} h_{0}^{2}\right)$ turns eddy diffusion into a vertical eddy viscosity (see e.g. Rhines and Young 1982, Olbers et al. 1985). One unit of $\tau$ in the above relation derives from the direct wind forcing (in the zonal momentum balance) and one half from Ekman pumping. In the 'baroclinic Stommel regime' the transport is thus independent of viscosity and increases quadratically with the Rossby radius and inversely with the diffusivity. Compared to the flat bottom regime discussed above, the transport in this high topography regime is extremely reduced: for the windstress (7) we get 887 in dimensionless units (三 $400 \mathrm{~Sv}$; this occurs at $\delta=0.25)$ which is a reduction by a factor of 8 compared to the flat bottom value 4990 . Note that the physics and equations in the suite of equations (25), (26) and (31) explain the vastly different ACC transports in the Bryan-Cox scenario.

\subsection{Numerical solutions}

In this section we discuss numerical solutions of the full coupled system in linearized form, gained by the MATLAB tool bvp4c.m. The relative accuracy is very high, usually $10^{-8}$, the grid is made by a couple of hundred points. The forcing is taken from (7), and we will consider purely wind-driven solutions (in which $Q_{0}=0$ ), and purely buoyancy-driven solutions (in which $\tau_{0}=0$ ). Furthermore, solutions for an artificial system are considered in which only the direct wind forcing is implemented in (9) but the Ekman pumping term in (20) is set to zero. In the buoyancy-driven cases the amplitude of the buoyancy forcing is chosen as $Q_{0}=\tau_{0} \lambda^{2} /(2 f)$ where $\tau_{0}$ is the amplitude of the wind driven cases. With the choice for meridional dependencies of the windstress and the buoyancy forcing these experiments thus present also the case of a system which is driven only by the Ekman pumping term.

Most of the solutions of the low-order model were repeated with the complete BARBI model. Examples are shown in Figure 1, and some of the transport values are inserted in Figure 4 . The linear solutions are very similar to those obtained from the low-order model discussed in this section. 
Solutions for the barotropic state The set of equations (9) to (14) describes a barotropic state if all $\phi \equiv 0$. The solution is exemplified in Figure 3. The most important signatures are the steering of the current by the $f / h$-contours and the drastic decay of the current amplitude and transport with increase of the topography height (for transport see the left panel of Figure 4). Notice also that the nonzonal components $\psi_{S, C}$ appear with large amplitudes almost spontaneously at non-zero topography heights; their effect on the transport is still small because of the $\eta$-factor in the formstress. They decay then with increasing heights but the corresponding formstress increases. A characteristic asymmetry develops in the current profiles at larger topographic height visible in the scaled profiles; it is due to the quadratic pressure correction $\eta P_{S}^{\prime} / 2$ in the geostrophic balance (12).

The balance of zonal momentum for the above barotropic state is displayed in the left set of panels of Figure 3 for the same topography heights as the profiles. The basic flat bottom balance between windstress and friction changes with increasing topography height to a balance between windstress and the barotropic bottom formstress $\eta P_{C} / 2$. Note that friction becomes very small at high topography but interestingly, it is not everywhere a sink of eastward momentum. The formstress is a brake for the current in the center, but acceleration occurs (though small) at high topography at the current flanks.

Linear baroclinic solutions We exemplify the baroclinic equations by a somewhat artificial system - a solution for a baroclinic state with suppressed barotropic variables, i.e. all $\psi$ are put to zero in the equations (20) to (22). Forcing is performed by $\mathcal{Q}=Q_{0} \cos y \sin ^{3} y$ and zero windstress, or equivalently via Ekman pumping with a windstress $\tau=\tau_{0} \sin ^{4} y$ with a corresponding amplitude. The $\mathcal{Q}$-forcing can be viewed as cooling in the southern part of the channel in balance and heating in the northern part. The result is shown in Figure 5. The purely baroclinic state has similarity to the barotropic one considered above: also $\phi$ follows $f / h$-contours. The shear transport $S_{0}=-\left(\phi_{0}+\eta \phi_{S}\right)^{\prime} / f$ arises by a balance between
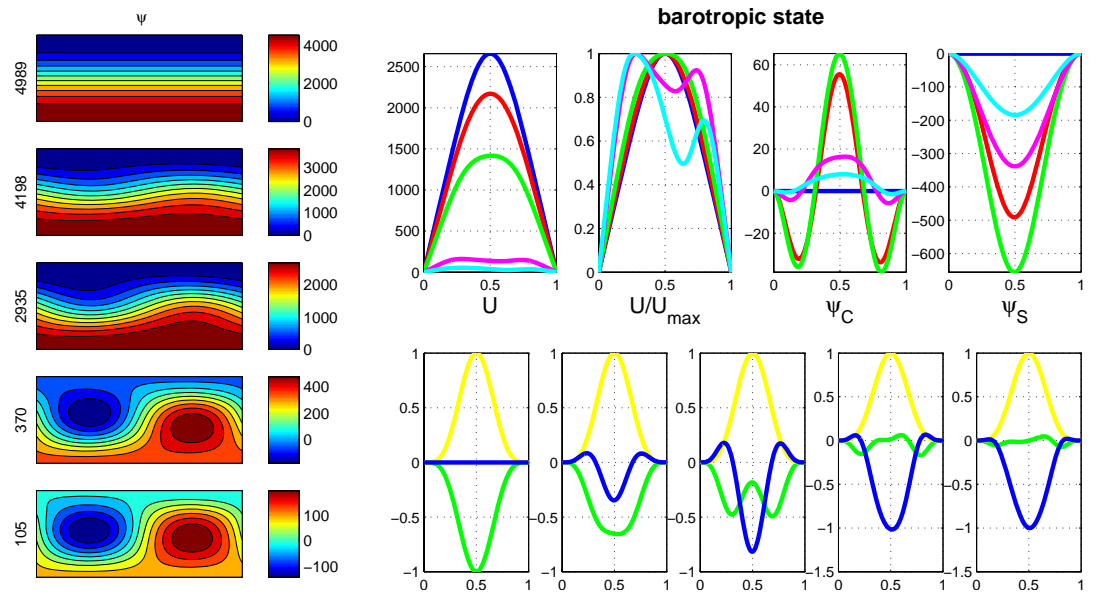

Figure 3: Solution of the model (9) to (14) for a barotropic state with wind forcing $\tau=\tau_{0} \sin ^{4} y$ and topography $\eta=\delta \sin ^{2} y$ with five heights $\delta=0,0.0125,0.025,0.125,0.25$. Left panels: streamfunction $\psi$ (the number on the left hand side is the dimensionless transport). Right panels: profiles and balance of zonal momentum for the corresponding heights. Line coloring for the balance terms: windstress [yellow], friction [green], formstress [blue]. 

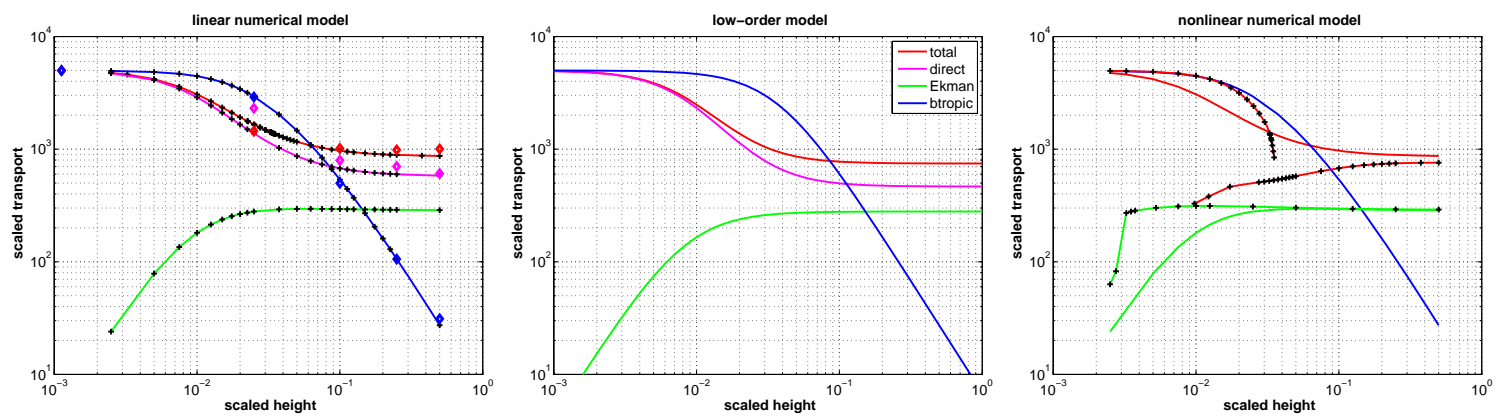

Figure 4: Left panel: transport for all linear linear wind-driven solutions obtained by numerical integration (indicated by crosses and connected by colored lines): barotropic state [blue], baroclinic state [red], direct wind forcing only [magenta], Ekman pumping or buoyancy forcing only [green]. The diamond symbols refer to numerical experiments with the full BARBI model. Middle panel: analytical solution according to the low order fit (38) discussed below. Right panel: transport for all nonlinear linear wind-driven and buoyancy-driven solutions (indicated by crosses and connected by colored lines). The linear solutions are included as colored lines.

diffusion $\kappa S_{0}$, the explicit forcing contained in (20) and a baroclinic vertical pumping term which is due to a baroclinic wave response of zonal shear flow forced over the topography. The balance shifts from forcing against diffusion for flat bottom towards forcing against pumping with higher topography. Notice that the baroclinic formstress has a profile which is similar to the barotropic one in the barotropic state but $U_{0}(y)$ and $S_{0}(y)$ differ substantially. As in the barotropic state, we also find the baroclinic transport to decrease with increasing height.

Linear coupled solutions The above described baroclinic state is artificial because the baroclinic formstress would accelerate a zonal mean current which in turn would excite a barotropic formstress feeding back on the baroclinic variables, as described in the model equations. Next we consider this complete system but still as a wind forced linear wave system. It is described by the coupled system (9) to (14) and (20) to (22) with $\gamma=0$. The windstress profile is as before, and baroclinic forcing is absent. The $S, C$-components in such a system represent two modes of stationary planetary-topographic Rossby waves as discussed above. The profiles and balance of the solutions are shown in Figure 6, the transport in Figure 4. Note that $f / h$-steering is absent in this regime. Also asymmetries have vanished from the zonal current profile. The barotropic wave component has decreased in amplitude: the current is thus almost zonal and drastically different from the barotropic state.

Furthermore, the decrease of the transports contained in $U_{0}$ and $S_{0}$ occurs faster at low height than the barotropic state: the coupling of the barotropic and baroclinic equations obviously introduces another decay scale (see Figure 4). We explain this feature in the next section. A remarkable difference to the purely barotropic state in Figure 3 appears in the balance of total momentum: the friction does not become as insignificant with increasing topography height as in the barotropic state and the dominant balance at large heights occurs now between windstress driving the eastward flow, aided by the barotropic formstress in the jet center, and baroclinic formstress acting everywhere as brake.

Likewise, the balance of the shear flow differs substantially from the baroclinic state: now 



Figure 5: Solution of the baroclinic equations (20) to (22) for a baroclinic state (i.e. $\psi \equiv 0$ ) with forcing by $\mathcal{Q}$-forcing with $\mathcal{Q}=Q_{0} \cos y \sin ^{3} y$ and topography $\eta=\delta \sin ^{2} y$ with five heights $\delta=0,0.0125,0.025,0.125,0.25$. Left panels: potential energy $\phi$. Right panels: profiles and balance of zonal shear flow for the corresponding heights. Line coloring for the balance terms: source term $\mathcal{S}$ [red], diffusion [green], formstress [magenta].

the baroclinic formstress drives the eastward shear flow, and the barotropic formstress and - with clear dominance - the density diffusion (GM viscosity) are westward accelerations.

Most important, however, is the increased level of transport at high topography (referring to Figure 4 ): at $\delta=0.25$ the transport in the barotropic state is 105, in the coupled state it is about 887. The current no longer tends to zero with increasing height of the topography but rather equilibrates at a non-zero high level which is in agreement with the topographic saturation scenario revealed in the last section.

\subsection{Linear topographic resistance}

Some important mechanisms that determine the channel transport can be gained from analyzing the steady linear wave response in more detail than presented in the above perturbation analysis. Eliminating the sine components $\psi_{S}$ and $\phi_{S}$ we derive after some minor approximations at

$$
\begin{aligned}
& F_{\text {trop }}=\frac{1}{2} f \eta \psi_{C}=-\frac{1}{2} \eta \frac{\left(\epsilon / \beta^{2}\right)}{(\epsilon / \beta)^{2} \mathcal{L}^{4}+1} \mathcal{L}^{2} \eta\left(U_{0}-S_{0}\right)=\mathcal{T}_{\text {trop }}\left(U_{0}-S_{0}\right) \\
& F_{\text {clin }}=-\frac{1}{2} \eta \phi_{C}=\frac{1}{2} \eta \frac{\left(\kappa \lambda^{2} / c^{2}\right)}{(\kappa / c)^{2} \mathcal{L}^{2}+1} \mathcal{L} \eta\left(U_{0}-S_{0}\right)=\mathcal{T}_{\text {clin }}\left(U_{0}-S_{0}\right)
\end{aligned}
$$

for the barotropic and baroclinic formstress components. The sign convention is here that a positive formstress is a source of eastward momentum. Evidently, bottom formstress 


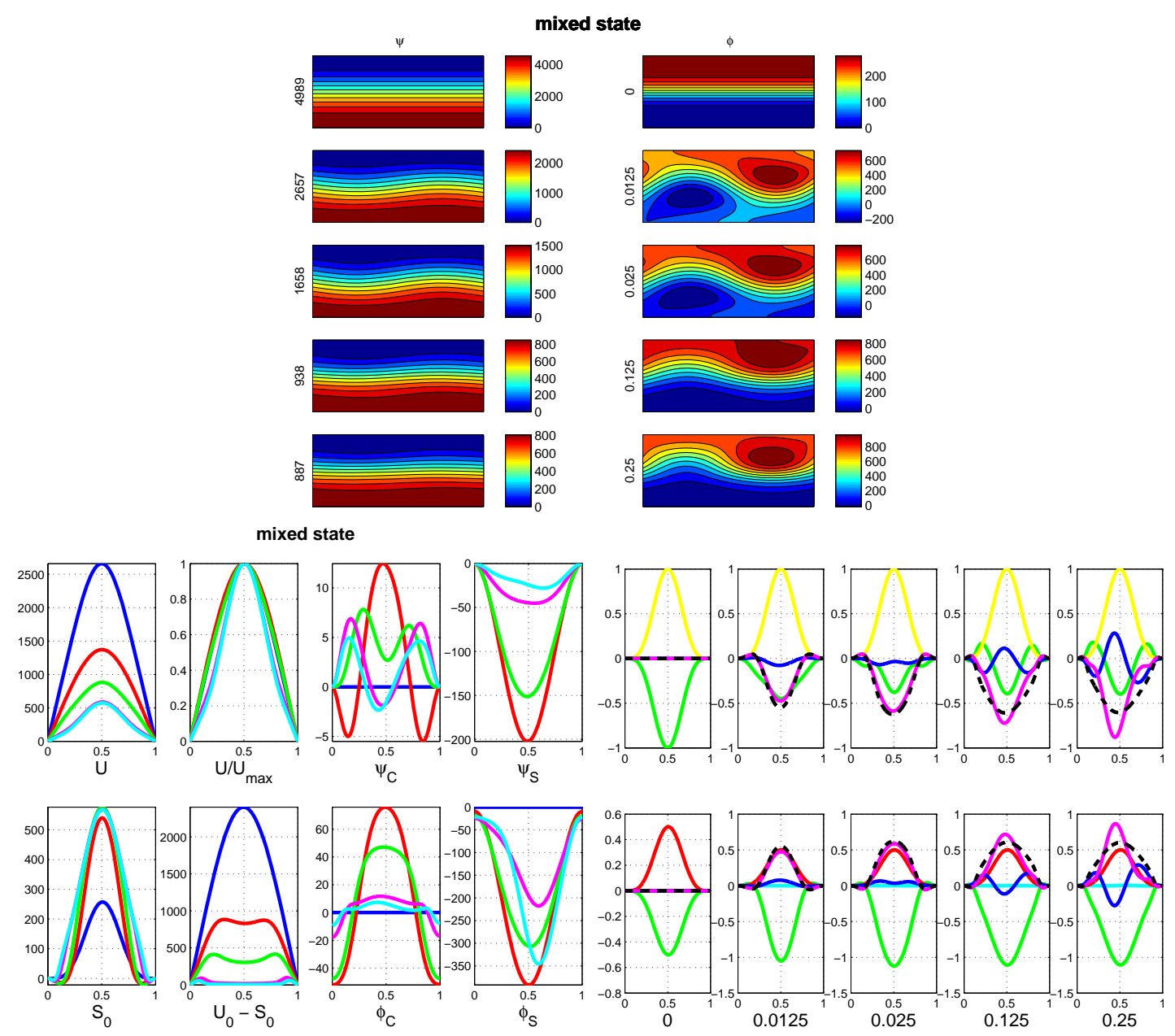

Figure 6: Upper panels: solution of the coupled equations (9) to (14) and the linearized versions of (20) to (22) with wind forcing $\tau=\tau_{0} \sin ^{4} y$ and topography $\eta=\delta \sin ^{2} y$ with five heights $\delta=$ $0,0.0125,0.025,0.125,0.25$. Lower panels: balance of zonal momentum and zonal shear flow for the corresponding heights. Line coloring for the balance terms of zonal momentum: windstress [yellow], friction [green], barotropic formstress [blue], baroclinic formstress [magenta], total formstress [black dashed]. For shear current: source term $\mathcal{S}$ [red], diffusion [green], barotropic formstress [blue], baroclinic formstress [magenta] total formstress [black dashed].

only builds up if topography is non-flat and if deep flow is present, which is here represented by $U_{0}-S_{0}$. The relation between the deep flow and the formstress components is mediated by 'topographic response operators' $\mathcal{T}_{\text {trop }}$ and $\mathcal{T}_{\text {clin }}$ for the barotropic and baroclinic cases, respectively. Notice that the formstress components are proportional to the respective viscosity/diffusion coefficients, $\epsilon$ for the barotropic one and $\kappa$ for the baroclinic one. In the barotropic formstress in (32) the first term in the denominator is very small, $(\epsilon / \beta)^{2} \sim 2.7 \times 10^{-5}$. In the baroclinic formstress in (32) it is also the second term which dominates since $(\kappa / c)^{2} \sim 5.6 \times 10^{-3}$ can be considered small but since $c^{2} \sim \lambda^{4}$, a decrease of the Rossby radius by only a factor 3 makes this ratio of order 1 . At such large values of $(\kappa / c)^{2}$ the baroclinic formstress varies as $\kappa^{-1}$. 
The sum of formstress components, $F=F_{\text {trop }}+F_{\text {clin }}$, operates in the barotropic momentum balance (17). Combining the two topographic response operators to $\mathcal{T}=\mathcal{T}_{\text {trop }}+\mathcal{T}_{\text {clin }}$ this balance reads

$$
-\epsilon U_{0}^{\prime \prime}=F+\tau=\mathcal{T}\left(U_{0}-S_{0}\right)+\tau
$$

and likewise, the baroclinic balance (23) becomes in linearized form

$$
\frac{\kappa}{\lambda^{2}} S_{0}=-F+\mathcal{S}=-\mathcal{T}\left(U_{0}-S_{0}\right)+\mathcal{S}
$$

The balances are coupled for non-zero topographic height, and either of $U_{0}$ or $S_{0}$ may be eliminated. Aiming at an equation for the zonal transport, we eliminate $S_{0}$ and find

$$
F=\mathcal{T}\left(U_{0}-S_{0}\right)=\frac{\mathcal{T}}{1-\frac{\lambda^{2}}{\kappa} \mathcal{T}}\left(U_{0}-\frac{\lambda^{2} \mathcal{S}}{\kappa}\right)
$$

The formstress response to the bottom layer transport $U_{0}-S_{0}$ is described by $\mathcal{T}$ but, notably, the response to the total transport $U_{0}$ involves a baroclinic feedback loop and this leads to the more complicated response operator given by the last relation in (35), involving the baroclinic forcing $\mathcal{S}$ as well. Then, finally, using these relations in the zonal barotropic balance (9) a complicated relation between the barotropic transport variable $U_{0}$ and the forcing is derived,

$$
\mathcal{R} U_{0}=\left[-\epsilon \frac{\partial^{2}}{\partial y^{2}}-\frac{\mathcal{T}}{1-\frac{\lambda^{2}}{\kappa} \mathcal{T}}\right] U_{0}=\tau-\frac{\mathcal{T}}{1-\frac{\lambda^{2}}{\kappa} \mathcal{T}} \frac{\lambda^{2} \mathcal{S}}{\kappa}=\mathcal{F}
$$

This equation is regarded here more as a symbolic frame rather than the integro-differential equation which it represents from a mathematical point of view. We will utilize the 'resistance relation' (36) in a qualitative way to discuss the dependence of transport on the applied forcing.

We refer to $\mathcal{R}$ as 'resistance operator' because it relates the current $U_{0}$ to the forcing $\mathcal{F}$ which is the sum of three contributions: the direct wind driving, the Ekman pumping, and baroclinic forcing. The latter two are contained in $\mathcal{S}$. Under the condition of the Southern Ocean windstress (being eastward) and the integrated buoyancy flux (cooling in the south and heating in the north) all forcing terms accelerate an eastward current. The direct forcing of zonal flow by the wind does not depend on the topography height $\delta$ whereas the contribution from Ekman pumping acting on the mean stratification, and likewise the buoyancy-forced part, build up quadratically with $\delta$ and approach a magnitude which gets independent of $\delta$ at some critical height (when the $\mathcal{T}$-part in the denominator overwhelms unity). Then we are in the saturated state described above.

The barotropic state is obtained as limit $\lambda^{2} / \kappa \rightarrow 0$. We obtain

$$
\mathcal{R} U_{0}=\left[-\epsilon \frac{\partial^{2}}{\partial y^{2}}-\mathcal{T}_{\text {trop }}\right] U_{0}=\tau
$$


which replaces the more qualitative estimate (26). Both contributions to the barotropic version of the resistance - the friction part and the barotropic formstress part - are proportional to the viscosity $\epsilon$. The barotropic pressure gets out of phase with the topography only because of friction. It seems worth noting that (9) to (14) imply an upstream shift of the streamfunction of order $\epsilon / \beta$. There is a pressure high upstream of the topography and a low downstream with respect to the topography elevation. Topographic resistance is seen to overcome friction when the topographic height $\delta$ exceeds $\delta_{\text {trop }}=\beta \ell$ where $\ell$ is some meridional length scale associated with the $U_{0}$-profile.

For this estimate we have replaced the operator by an algebraic expression, $\mathcal{T}_{\text {trop }} \sim$ $-\left(\epsilon / 2 \ell^{2}\right)\left(\delta / \delta_{\text {trop }}\right)^{2}$. Correspondingly, we find $\mathcal{T}_{\text {clin }} \sim-\left(\epsilon / 2 \ell^{2}\right)\left(\delta / \delta_{\text {clin }}\right)^{2}$ where the critical topographic height for the baroclinic response is $\delta_{\text {clin }}=(1 / 2) \beta \lambda \sqrt{\epsilon / \kappa}$ for small $(\kappa / c)^{2}$, and $\delta_{\text {clin }}=\sqrt{\epsilon \kappa} /\left(\lambda \ell^{2}\right)$ for a large $(\kappa / c)^{2}$. For $\ell=1.15$ (see below for this setting) and our standard parameter values we find $\delta_{\text {trop }} \sim 0.06$ and $\delta_{\text {clin }} \sim 0.01$. Consequently, the baroclinic decay height scale is almost an order of magnitude smaller than the critical height scale of a barotropic system. The barotropic height scale is thus completely masked in the full resistance function as clearly visible in Figure 4.

Notice that $\delta_{\text {clin }}^{2}$ is a Prandtl number (or an inverse Rayleigh number for large $(\kappa / c)^{2}$ ) whereas $\delta_{\text {trop }}$ is associated with a ratio of flow scale to planetary scale. A third scale $\delta_{\star}$ derives from the operator $\lambda^{2} \mathcal{T} / \kappa$ in the denominator in (36). With $\mathcal{T} \sim T_{\text {clin }}$ and our standard parameters we get $\delta_{\star} \sim c \ell / \lambda^{2}=\delta_{\text {trop }} / 2 \sim 0.03$.

Expressing the meridional operators $\partial^{2} / \partial y^{2}$ and $\mathcal{L}$ in the above relations by the scale $\ell$, results in a primitive form of a low-order model, but still one which is sufficient for a qualitative discussion. The resistance and the forcing terms of the zonal current are now written very roughly as functions of the topography height and the resistance and forcing terms of $\mathcal{R} U_{0}=\mathcal{F}$ become

$$
\mathcal{R} \cong \frac{\epsilon}{\ell^{2}}\left[1+\frac{1}{2} \frac{d \delta^{2}}{1+\frac{1}{2} \frac{\epsilon \lambda^{2}}{\ell^{2} \kappa} d \delta^{2}}\right] \quad \mathcal{F} \cong \tau+\frac{1}{2} \frac{\epsilon}{\ell^{2}} \frac{d \delta^{2}}{1+\frac{1}{2} \frac{\epsilon \lambda^{2}}{\ell^{2} \kappa} d \delta^{2}} \frac{\lambda^{2} \mathcal{S}}{\kappa}
$$

with $d=\vartheta^{2} / \delta_{\text {trop }}^{2}+\vartheta / \delta_{\text {clin }}^{2}$. Each $\mathcal{L}$ has been replaced by $-\vartheta / \ell^{2}$. The factor $\vartheta$ is roughly of order $1+\ell^{2} b^{2}$. We do not attribute any weight into the $y$-dependence of this relation - it is meant in some integral fashion. The transport for the flat bottom is $U_{f b}=\tau \ell^{2} / \epsilon$. To get the correct transport value for the forcing described in (25) we have to put $\ell^{2}=\left(15+4 \pi^{2}\right) \pi / 128$ or $\ell \sim 1.15$.

The inverse resistance function which is involved in the transport $U_{0}=\mathcal{R}^{-1} \mathcal{F}$ decays quadratically at small $\delta$, under baroclinic condition much faster than under a barotropic one (for the standard parameters where we find $\delta_{\text {trop }} \gg \delta_{\text {clin }}$ ). In a barotropic state (i.e. $\lambda=0$ ) the transport approaches zero with increasing height with this $\delta^{-2}$ dependence (of course the functions become meaningless for $\delta \geq 1$ ). But under baroclinic conditions (i.e. $\lambda \neq 0$ ), even if only forcing by wind is considered, the response becomes more complicated because of the baroclinic feedback mentioned above in (35) which leads to denominators in (38). Now the transport dependence on $\delta$ runs into a plateau at large heights $\delta \gg \delta_{\star}$. If the system is forced by $\mathcal{S}$ alone (Ekman pumping or baroclinic forcing), the forcing itself builds up as $\delta^{2}$ at low heights and turns to a plateau at high $\delta \gg \delta_{\star}$, consistent with the numerical results of Figure 4 . In this 'saturated' range of topography heights the transport is determined by the 'baroclinic Stommel' equation (31). The resistance relation for this state can be obtained without 
access to the highly approximated form (38): assuming a large $\mathcal{T}$ in (36) we immediately find (30).

\section{Nonlinear theory}

The nonlinear terms in the baroclinic balances (20) to (22) are

$$
\begin{aligned}
& \mathcal{A}_{0}=-\left(\psi_{S} \phi_{C}-\psi_{C} \phi_{S}\right)^{\prime}+\eta\left[\psi_{C}\left(\phi_{0}+\eta \phi_{S}\right)^{\prime}+U_{0} \phi_{C}\right] \\
& \mathcal{A}_{C}=-\psi_{S}\left(\phi_{0}+\eta \phi_{S}\right)^{\prime}-U_{0}\left(\phi_{S}+2 \eta \phi_{0}\right) \\
& \mathcal{A}_{S}=\psi_{C}\left(\phi_{0}+\eta \phi_{S}\right)^{\prime}+U_{0} \phi_{C}
\end{aligned}
$$

Implementing $\mathcal{A}_{0}$ in the zonal balance (20) results in the standing eddy interfacial stress components in the baroclinic balances (23) and (24), given by

$$
\mathcal{N}_{0}=\frac{\gamma}{2 f \lambda^{2}} \int_{0}^{y} \mathcal{A}_{0} d y^{\prime}=-\frac{\gamma}{2 f \lambda^{2}}\left[\left(\psi_{S} \phi_{C}-\psi_{C} \phi_{S}\right)+\int_{0}^{y} \eta\left(S_{0} f \psi_{C}-U_{0} \phi_{C}\right) d y^{\prime}\right]
$$

It consists of a wave-wave interaction part and wave-mean-flow interaction part. With $\mathcal{A}_{C, S}$ inserted the two wave balances become

$$
\begin{aligned}
-\kappa \mathcal{L} \phi_{C}+\left(\frac{1}{2} \gamma U_{0}-c\right)\left(\phi_{S}+2 \eta \phi_{0}\right) & =\lambda^{2} \eta\left(U_{0}-S_{0}\right)+\frac{1}{2} \gamma S_{0} f \psi_{S} \\
-\kappa \mathcal{L}\left(\phi_{S}+2 \eta \phi_{0}\right)-\left(\frac{1}{2} \gamma U_{0}-c\right) \phi_{C} & =-\frac{1}{2} \gamma S_{0} f \psi_{C}
\end{aligned}
$$

The wave-mean flow interaction thus introduces zonal advection of the specific wave component and a source term.

Standing eddy interfacial formstress Simplifying the operators to an algebraic form as in the previous section, we can estimate the size of the interfacial formstress associated $\mathcal{N}_{0}$. Inserting the flat bottom relations $\psi_{S}=-(\beta / \epsilon) \mathcal{L}^{-2} \psi_{C}$ and $\phi_{S}=-(c / \kappa) \mathcal{L}^{-1} \phi_{C}$ into the wave-wave part yields

$$
-\frac{\gamma}{2 f \lambda^{2}}\left(\psi_{S} \phi_{C}-\psi_{C} \phi_{S}\right)=\delta^{2} \frac{\alpha_{w w}}{\delta_{\text {trop }}^{2} \delta_{c l i n}^{2}}\left(U_{0}-S_{0}\right)^{2}
$$

with a negative $\alpha_{w w}=-\gamma \beta \epsilon \vartheta / 2 \lambda^{2}$. In this simplified approximation the wave-wave part is always a downward transport of zonal momentum. Likewise, the wave-mean flow part becomes

$$
-\frac{\gamma}{2 f \lambda^{2}} \int_{0}^{y} \eta\left(S_{0} f \psi_{C}-U_{0} \phi_{C}\right) d y^{\prime}=\delta^{2} \frac{\alpha_{w m}}{\delta_{\text {trop }}^{2} \delta_{\text {clin }}^{2}}\left(\vartheta \delta_{\text {clin }}^{2} S_{0}+\delta_{\text {trop }}^{2} U_{0}\right)\left(U_{0}-S_{0}\right)
$$

with a negative $\alpha_{w m}=\gamma \epsilon /\left(2 f \lambda^{2} \ell\right)$. This part is thus a downward transport if $U_{0}>0$ and $U_{0}-S_{0}>0$ (assuming $U_{0} \delta_{\text {trop }}^{2}>S_{0} \delta_{\text {clin }}^{2}$ as in our standard parameter set). All numerical solutions shown in Figure 7 have a downward standing eddy flux. 
Supercritical flow The flat bottom wave speed $c$ in the baroclinic balances (21) and (22) is now replaced by $c_{U}=\frac{1}{2} \gamma U_{0}-c$ which measures the supercriticality of the current: in general $c_{U}>0$, so baroclinic waves no longer propagate westward but are swept eastward by the current. For our standard parameters this is the case if $U_{0}$ is above 130 . All numerical solutions shown in Figure 7 are thus supercritical.

It is interesting to note that in the limit of small diffusion, $\kappa \rightarrow 0$, the balance (42) implies

$$
\begin{gathered}
F_{\text {clin }}=-\frac{\frac{1}{2} \gamma S_{0}}{\frac{1}{2} \gamma U_{0}-c} F_{\text {trop }} \\
F=F_{\text {clin }}+F_{\text {trop }}=\frac{\frac{1}{2} \gamma\left(U_{0}-S_{0}\right)-c}{\frac{1}{2} \gamma U_{0}-c} F_{\text {trop }}
\end{gathered}
$$

which should govern the sign relations between the barotropic, baroclinic and total formstress components; e.g. for supercritical flow and eastward $S_{0}$ the barotropic and baroclinic formstress components must oppose each other. In a broad brush view the relations are satisfied in the numerical solutions described below.

Numerical solutions The full model exhibits a peculiarity which often occurs in nonlinear systems of low dimension. We find multiple steady states for identical forcing and system parameters. Figure 4 (right panel) reveals in the wind-driven case a fold bifurcation at a critical height $\delta_{c}$ (for the standard set of parameters $\delta_{c} \sim 0.035$ ). While the upper branch continues to exist for $\delta \rightarrow 0$ we found no stable solutions in the lower branch beyond a lowest height $\delta \sim 0.01$. The solutions for height below the bifurcation point are briefly described but not presented. The dynamical balances of the two stable solutions occurring at $\delta<\delta_{c}$ are drastically different. The balance of solutions with high zonal transport (upper branch) is dominantly between the windstress and the barotropic formstress when the height approaches the critical value, and the baroclinic transport is driven by the barotropic formstress and the Ekman pumping against the interfacial formstress due to the standing eddies. Approaching the bifurcation, the current shows increased tendency to follow $f / h$ contours. In the solution of the lower branch, having a smaller zonal transport but being more zonal than the upper branch, we find a negligible barotropic formstress; the baroclinic formstress takes the role of opposing the windstress. It also drives the baroclinic transport. In both branches, however, nonlinear terms - the standing eddy interfacial formstress - are the dominant sink of eastward baroclinic transport. They also play a major role in the baroclinic nonzonal equations.

At high topography, $\delta>\delta_{c}$, we found only one stable solution (depicted in Figure 7). The flow of these cases is nearly zonal (but the baroclinic field $\phi$ is clearly not zonal as its gradients are needed in JEBAR to overcome the $f / h$ Jacobian term). Remarkable changes occur in the dependence of the dynamical balances with increasing height. In the zonal barotropic balance the baroclinic formstress changes from a sink of eastward momentum (at $\delta \cong \delta_{c}$ ) to a complicated profile at high topography which presents a source over most of the current center and sinks on its flanks. The barotropic formstress develops from a marginal size (at $\delta \cong \delta_{c}$ ) to a profile which opposes the baroclinic one to a large degree. A corresponding transition takes place in the baroclinic zonal balance, however, for obvious reasons with interchanged signs for the barotropic and baroclinic formstress components. 

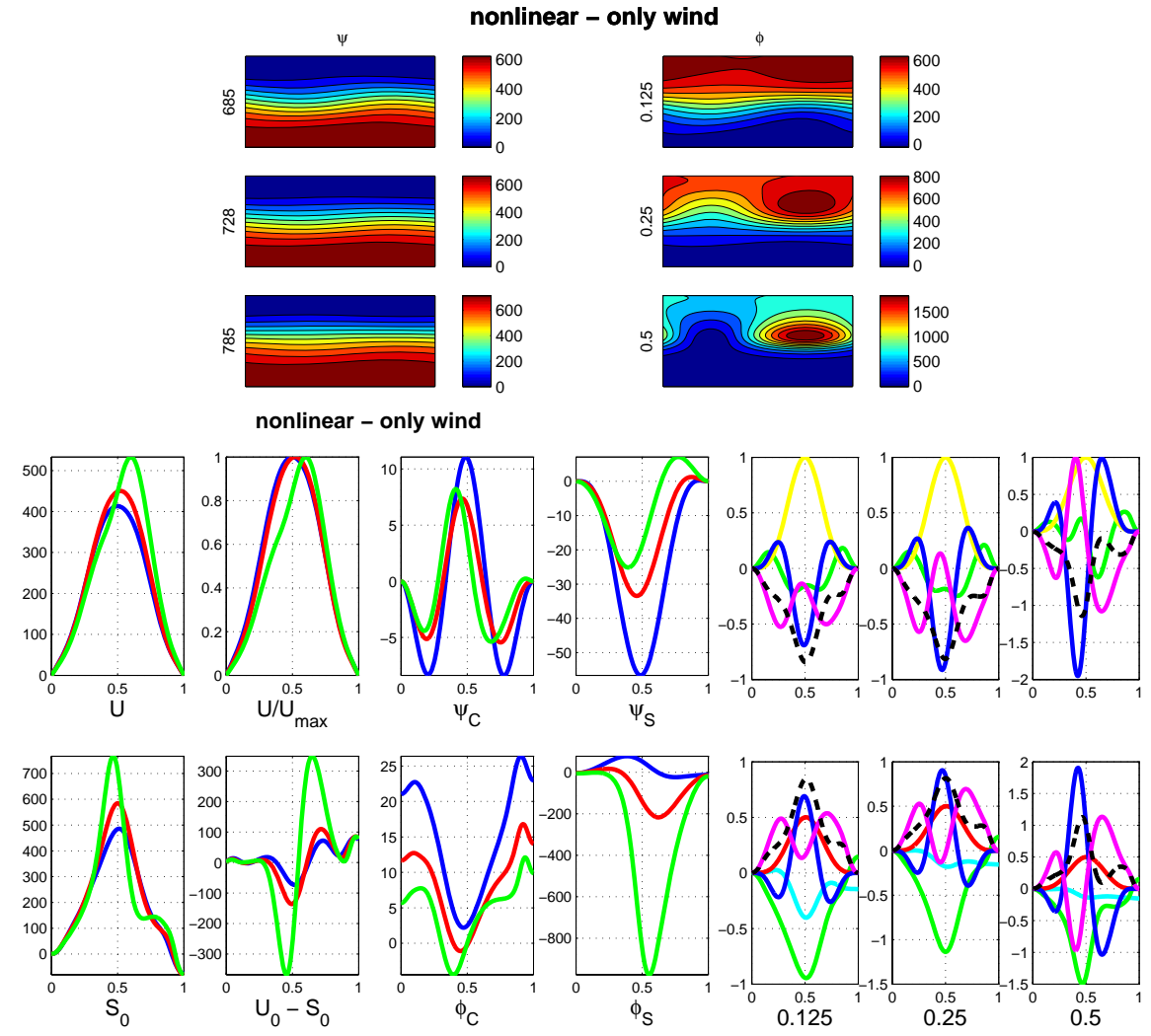

Figure 7: Nonlinear solutions for high topography. Upper panels: solution of the coupled full equations (9) to (14) and (20) to (22) with wind forcing $\tau=\tau_{0} \sin ^{4} y$ and topography $\eta=\delta \sin ^{2} y$ with five heights $\delta=0.0375,0.05,0.125,0.25,0.5$. Lower panels: balance of zonal momentum and zonal shear flow for the corresponding heights. Line coloring as in Figure 6.

The nonlinearity in the zonal baroclinic balance becomes marginal in this transition while it grows to a significant player in both baroclinic nonzonal equations (not shown).

Figure 4 (right panel) also shows the transport dependence on $\delta$ resulting from purely baroclinic forcing (or Ekman pumping). As in the linear solutions (left panel), the currents are much weaker than the directly wind-driven cases (for a $\mathcal{Q}$-amplitude which is of the size of a corresponding Ekman pumping). Comparison with the linear wind and buoyancy-driven solutions, and it becomes evident that nonlinearity increases the winddriven transport at subcritical heights (in the upper branch) but leads to a decrease at supercritical heights. The nonlinear buoyancy driven transport lies above the linear solution everywhere but approaches the linear values at high topography.

Important differences to the linear solutions are visible in the profiles of the baroclinic zonal transport $S_{0}$, or equivalently the gradient of potential energy. The profiles become more structured, and a small but dynamically important difference is the non-zero value at the northern boundary. It represents a flux of potential energy out of the system, revealing that there is an equivalent gain from the kinetic energy, which is induced by lifting the density stratification by the flow over the topography (see Appendix C).

The described bifurcations do not occur in the same way in numerical solutions of the 
complete BARBI model applied to the channel geometry, and hence they may be an artifact of the truncation (as often in truncated low-order models). With this in mind we deem it advisable to regard the above solutions for low and intermediate heights more as a mathematical dynamical system rather than applicable to a real physical system.

Nonlinear topographic resistance In the nonlinear theory the barotropic formstress remains as derived in (32) but the balances of $\phi_{C}$ and $\phi_{S}$ have significant nonlinear contributions. Eliminating $\phi_{S}$ results in the nonlinear version of the baroclinic formstress. With $\mathcal{L}^{2} \psi_{S}=-(\beta / \epsilon) \psi_{C}$ from (19) and some minor manipulations we arrive at

$$
F_{\text {clin }}^{n l} \cong \frac{1}{2} \frac{1}{\kappa^{2} \mathcal{L}^{2}+c_{U}^{2}}\left[\kappa \lambda^{2} \eta \mathcal{L} \eta-\gamma S_{0}\left(c_{U}+\frac{\kappa \beta}{\epsilon} \mathcal{L}^{-1}\right) \mathcal{T}_{\text {trop }}\right]\left(U_{0}-S_{0}\right)
$$

Still, as in the linear case, a non-zero formstress requires a non-zero bottom flow. We now have to solve the two nonlinear equations

$$
\begin{aligned}
-\epsilon U_{0}^{\prime \prime} & =\mathcal{T}_{\text {trop }}\left(U_{0}-S_{0}\right)+F_{\text {clin }}^{n l}\left[U_{0}, S_{0}\right]+\tau \\
-\epsilon U_{0}^{\prime \prime}+\frac{\kappa}{\lambda^{2}} S_{0} & =\mathcal{N}_{0}\left[U_{0}, S_{0}\right]+\tau+\mathcal{S}
\end{aligned}
$$

for $U_{0}$ and $S_{0}$. Reduction of this nonlinear system to a low-order algebraic form is straightforward but not very successful. The physical setting is completely analogous to the barotropic Charney-DeVore model (Charney and DeVore 1979), however, the three terms in the bracket of (46) are competing for the sign of the formstress $F_{\text {clin }}^{n l}$ (the first two are negative, the third is positive and generally the largest) which makes it difficult to generate a quantitatively correct algebraic low-order model. Topographic resonance can be achieved but detailed comparison with the numerical solutions becomes questionable. Of course, without the knowledge from the numerical solutions this failure would pass unnoticed.

\section{Summary and conclusions}

The most important properties of the dynamical balance of the ACC can be understood by means of the reduced physics model BARBI which embraces the main components of the interaction of the barotropic and baroclinic subsystems of ocean dynamics which are relevant in circumpolar flows. The BARBI model, derived in Olbers and Eden (2003), uses an unusual representation of the vertical structure of baroclinic flow. In the version used in this study only two variables are considered, referring to the barotropic and first baroclinic modes. The dynamical variables are the vertically integrated momentum and the baroclinic potential energy of the entire water column. The model describes the interaction of the two lowest modes of planetary-topography Rossby waves and includes diabatic processes diffusion of momentum and vorticity - and advection of density by the zonal current and by eddies. It applies to topography of arbitrary height and conserves momentum, vorticity and total energy. Wind forcing appears directly in the momentum balance and indirectly as Ekman pumping - in the potential energy balance. The latter also has an explicit forcing from interior diapycnal mixing. 
Configured in a simple reentrant channel geometry with sinusoidal topography a loworder model is derived which makes it possible to determine the circulation and channel transport in the presence of topography by analytical means. We have solved this model by successive approximations: expansion into zonal modes, explicit numerical integration of the dynamical equations of the coupled Rossby waves to get the meridional dependence of the modal amplitudes, and - finally - a quite crude reduction to an algebraic set of equations which determines the transport as function of the applied wind stress, buoyancy forcing, and the system parameters, notably the height of the topography and the coefficients for viscosity and eddy advection. Apart from the forcing, the fundamental variables governing the transport are the bottom formstress and eddy-induced diffusivity of density advection. These features are operating in all numerical simulations of the ACC (see e.g. the OGCM simulations of Cai and Baines 1996, Gent et al. 2001, and the BARBI simulations of Olbers et al. 2006). The influence of eddy advection of density was particularly considered in models which use the Gent-McWilliams parameterization (e.g. Gent et al. 2001).

Different processes are conceivable to generate bottom formstress. In this study we consider the process which is inherent in the fluid dynamical conservation laws also in the absence of any external effects: the generation and locking of Rossby waves by topography, which then set up pressure differences across the submarine topographic barriers. Formstress is determined by the component of pressure that is out-of-phase with the topography in the zonal direction.

Topographically induced resistance is proposed in this study as a new concept to describe how the bottom formstress is generated by planetary-topographic Rossby waves. With two wave modes present there are two contributions to the formstress: the barotropic component is locked to the topography by viscous effects, and the baroclinic component relates to the Gent-McWilliams diffusivity. The wave induced pressure field generally builds up a westward acceleration and by and large resists against the implied eastward forcing. We derive expressions for the critical heights for the different resistance contributions. With sufficiently high topographic barriers and stratification present, the baroclinic resistance is most important. Density advection adds nonlinearity to the wave system, but the resistance concept stays intact: the baroclinic resistance becomes a nonlinear function of the zonal current, and multiple equilibria might occur much along the ingredients of the Charney-DeVore system (Charney and DeVore 1979, see Olbers and Völker 1996 and Völker 1999 for a baroclinic ACC version). We show that in a supercritical current, i.e. when the current speed exceeds the speed of baroclinic Rossby waves, the formstress components tend to increase with topography height but almost compensate each other (their difference still has to balance the applied windstress).

The wave-induced topographic resistance and viscous braking - the frictional resistance - emerge in the zonal balance of the channel flow and determine the zonal transport in a relation similar to Ohm's law: resistance times transport equals the applied forcing. The resistance, however, is a complicated differential-integral operator depending on the systems parameters, in particular the topography height, and its action on the transport is not everywhere (at all latitudes) positive. Our analysis reveals how the different contributions from windstress, Ekman pumping and buoyancy forcing add to the total forcing. With our standard parameters and forcing amplitudes the direct wind forcing is clearly dominant in generating zonal transport. Forcing by Ekman pumping and diapycnal mixing come only into play with increasing topographic height (there is no effect from them for a flat bottom ocean). 
With increasing height of the topography the coupled forced wave system runs into a saturated state in which baroclinicity takes over the control. Then the influence of the $f / h$ characteristics is wiped out, the flow becomes almost zonal and the transport almost independent of the height of topography. The critical height for this state is $\pi h_{0}(\beta /|f|) B_{y}^{2} / L_{x} \sim$ $1000 \mathrm{~m}$, where $B_{y}$ is a meridional length scale of the flow, $L_{x}$ a zonal wavelength of the topography and $h_{0}$ the mean water depth. We refer to this saturated state as the 'baroclinic Stommel regime' because the circulation and transport is governed by a vorticity balance having similarity to Stommel's equation for a wind-driven gyre over a flat bottom. In the present regime, however, bottom friction is replaced by the lateral eddy diffusivity converted to a vertical viscosity, e.g. $K f^{2} / N^{2}$, and forcing is the above mentioned triad of direct windstress, Ekman pumping and diapycnal mixing. Viscous effects are minor for the transport but still necessary to arrest the formstress induced by the barotropic Rossby wave. This state is described for more realistic ACC simulations by Olbers et al. (2006).

In summary, the low-order model developed in this study explains in a satisfactory and consistent way the essential features of the numerical simulations of the ACC discussed in the introduction, referred to as the Bryan-Cox scenario.

Acknowledgements We appreciate numerous discussions with Christoph Völker and Sergey Danilov.

\section{References}

Borowski, D. The Antarctic Circumpolar Current: Dynamics of a circumpolar channel with blocked geostrophic contours. PhD thesis, University Bremen, 2003.

Borowski, D., Gerdes, R., and D. Olbers. Thermohaline and wind forcing of a circumpolar channel with blocked geostrophic contours. J. Phys. Oceanogr., 32:2520-2540, 2002.

Bryan, K. and M.D. Cox. The circulation of the World Ocean : a numerical study. Part I, A homogeneous model. J. Phys. Oceanogr., 2:319-335, 1972.

Cai, W., and P.G. Baines. Interactions between thermohaline- and wind-driven circulations and their relevance to the dynamics of the Antarctic Circumpolar Current, in a coarse-resolution global ocean general circulation model. J. Geophys. Res., 101:14073-14094, 1996.

Charney, J.G. and J.G. DeVore. Multiple flow equilibra in the atmosphere and blocking. J. Atmos. Sciences, 36:1205 - 1216, 1979.

Cox, M.D. A baroclinic numerical model of the Word Ocean: Preliminary Results. In Numerical Models of Ocean Circulation, pages 107-120, Durham, New Hampshire, Oct. 17 - 20, 1972, 1975.

Gent, P.R., W.G. Large, and F.O. Bryan. What sets the mean transport through Drake Passage? J. Geophys. Res., 106:2693-2712, 2001.

Gnanadesikan, A., and R. W. Hallberg. On the relationship of the Circumpolar Current to Southern Hemisphere winds in coarse resolution ocean models. J. Phys. Oceanogr., 30:2013-2034, 2000.

Krupitsky, A., and M.A. Cane. On topographic pressure drag in a zonal channel. J. Mar. Res., 52:1 23, 1994.

MacCready, P., and P.B. Rhines. Meridional transport across a zonal current: topographic localization. J. Phys. Oceanogr., 2001.

Munk, W.H., and E. Palmén. Note on dynamics of the Antarctic Circumpolar Current. Tellus, 3:53-55, 1951.

Olbers, D, and C. Eden. A simplified general circulation model for a baroclinic ocean with topography. Part I: Theory, waves and wind-driven circulations. J. Phys. Oceanogr., 33:2719-2737, 2003. 
Olbers, D., and C. Völker. Steady states and variability in oceanic zonal flows. In D.L.T. Anderson and J. Willebrand, editor, Decadal climate variability dynamics and predicition, pages 407-443, Berlin, 1996. Springer-Verlag.

Olbers, D., Borowski, D., Völker, C., and J.-O. Wolff. The dynamical balance, transport and circulation of the Antarctic Circumpolar Current. Antarctic Science, 16(4):439-470, 2004.

Olbers, D., Lettmann, K., and R. Timmermann. Six circumpolar currents - on the forcing of the Antarctic Circumpolar Current by wind and mixing. Ocean Dynamics, 57(DOI 10.1007/s10236006-0087-9):12-31, 2006.

Olbers, D.J., Wenzel, M., and J. Willebrand. The inference of North Atlantic circulation patterns from climatological hydrographic data. Reviews of Geophysics, 23(4):313-356, 1985.

Rhines, P. The dynamics of unsteady currents. In E. Goldberg, editor, The Sea, Vol. VI, pages 189-318, New York, 1977. Wiley.

Rhines, P.B. and W.R. Young. A theory of the wind-driven circulation. I. Mid-Ocean gyres. J. Mar. Res., 40 (Suppl.):559-596, 1982.

Rintoul, S.R., C. Hughes and D. Olbers. The Antarctic Circumpolar Current system. In G. Siedler, J. Church and J. Gould, editor, Ocean Circulation and Climate, pages 271-302, New York, 2001. Academic Press.

Sarkisyan, A.S., and V.F. Ivanov. Joint effect of baroclinicity and bottom relief as an important factor in the dynamics of sea currents. Akad. Nauk. Atmosph. Oceanic Phys., 7(2):173-188, 1971.

Stommel, H. A Survey of Ocean Current Theory. Deep-Sea Res., 4:149-184, 1957.

Tansley, C.E., and D.P. Marshall. On the dynamics of wind-driven circumpolar currents. J. Phys. Oceanogr., 31:3258-3273, 2001.

Völker, C. Momentum balance in zonal flows and resonance of baroclinic Rossby waves. J. Phys. Oceanogr., 29:1666-1681, 1999.

Walsteijn, F. Numerical methods for quasigeostrophic turbulence. With application to the Antarctic Circumpolar Current. Technical report, Institute for Marine and Atmospheric Research, Utrecht, R96-12, 1996.

Wang, L., and R.X. Huang. A linear homogeneous model of wind-driven circulation in a $\beta$-plane channel. J. Phys. Oceanogr., 25:587 - 603, 1995.

Wolff, J.-O., E. Maier-Reimer and S. Legutke. The Hamburg Ocean Primitive Equation Model. Technical Report No. 13, Modell Betreuungsgruppe (Ed.), 110 pp., ISSN 0940-9327. Technical report, German Climate Computer Center (DKRZ), 1997.

\section{Appendix}

\section{A The BARBI model and scaling}

Our model is a two-mode version of the reduced BARBI physics (BARotropic-BaroclinicInteraction ocean model, Olbers and Eden 2003) and can be summarized by the set of equations

$$
\begin{aligned}
\frac{\partial \mathbf{U}}{\partial t}+f \mathbf{k} \times \mathbf{U} & =-h \boldsymbol{\nabla} P-\nabla h^{2} \phi+\boldsymbol{\tau}+A_{h} \nabla^{2} \mathbf{U} \\
\frac{\partial \tilde{\mathbf{u}}}{\partial t}+f \mathbf{k} \times \tilde{\mathbf{u}} & =\frac{1}{3} h^{2}\left[\nabla h^{2} \phi-\tau\right] \\
\boldsymbol{\nabla} \cdot \mathbf{U} & =0 \\
\frac{\partial}{\partial t} h^{2} \phi+h \mathbf{U} \cdot \nabla \phi-\frac{1}{2} N^{2} \boldsymbol{\nabla} \cdot\left[\tilde{\mathbf{u}}+\frac{1}{3} \mathbf{U} h^{2}\right] & =\mathcal{Q}-\mathcal{D}[\phi]+K \nabla^{2} h^{2} \phi
\end{aligned}
$$


with $\tilde{\mathbf{u}}=\mathbf{u}_{2}^{\prime}, \phi=E_{1} / h^{2}$ in the notation of Olbers and Eden (2003). Equation (48) is the vertically integrated balance of momentum with a simplified viscous term. We will refer to the vertically integrated velocity

$$
\mathbf{U}=\int_{-h}^{0} \mathbf{u} d z
$$

as transport velocity but frequently also as transport; $h$ is the ocean depth, $P$ is the bottom pressure, and $\tilde{\mathbf{u}}$ is the second vertical moment of the baroclinic velocity $\mathbf{u}-\mathbf{U} / h$. In the low-order model the baroclinic momentum balance (49) is taken diagnostically to eliminate gravity waves. The mass balance (50) allows to represent $\mathbf{U}$ by the streamfunction of the volume transport, $\mathbf{U}=\mathbf{k} \times \nabla \psi$. Finally, equation (51) is the balance of baroclinic potential energy

$$
h^{2} \phi=g \int_{-h}^{0} z \rho d z
$$

where $\rho$ is a perturbation density about a mean background stratification (and scaled by a constant reference density) described by the Brunt-Väisälä frequency $N$. The latter is taken constant in this study. Evidently, the quantity $\phi$ can be associated with a dominating isopycnal depth. The potential energy balance has a prescribed source function $\mathcal{Q}$, and $\mathcal{D}[\phi]$ represents dissipation. The diffusive term in (51) together with the dissipation can be derived from a Gent-McWilliams parameterization of the eddy-induced density advection. This is outlined in Olbers et al. (2006).

We like to point out that most terms in the above balances result exactly by vertical integration of the primitive equations. However, the BARBI system has a closure to utilize $\mathbf{U}, E$ and $\tilde{\mathbf{u}}$ as a complete set of state variables. The closure mainly concerns the baroclinic pressure term in (49). For details we refer to Olbers et al. (2006) and only mention here that the Brunt-Väisälä frequency in (51) is an effective one related to the true one $N_{0}$ by $N_{0}=\pi N / \sqrt{6}$. The relation ensures that the first baroclinic Rossby radius $N h /|f| / \sqrt{6}$ of the above system equals the familiar form $N_{0} h /(|f| \pi)$. This actually is the BARBI closure.

A zonal channel is considered of length $L$ and constant width $B$ in the coordinate domain $0 \leq x \leq L$ and $0 \leq y \leq B$ on a $\beta$-plane. The BARBI system (48) to (51) is used in a dimensionless form. Scaling is performed by defining dimensionless quantities (denoted by an asterisk). We take $(x, y)=(B / \pi)\left(x^{*} / b, y^{*}\right), t=t^{*} /\left(b\left|f_{0}\right|\right), h=h_{0} h^{*}, f=\left|f_{0}\right| f^{*}$ and introduce the scale factors for the forcing functions, $\tau=T_{0} b \tau^{*}, \mathcal{Q}=Q_{0} b \mathcal{Q}^{*}$ where $b=2 B / L$ is the channel aspect ratio. Furthermore, scaling of the of streamfunction and potential energy is done by $\psi=\Psi_{0} \psi^{*}, \phi=\Phi_{0} \phi^{*}$ with $\Psi_{0}=T_{0} B /\left(\pi\left|f_{0}\right|\right)$ and $\Phi_{0}=T_{0} B /\left(\pi h_{0}^{2}\right)$, and pressure $P=P_{0} P^{*}$ by $P_{0}=T_{0} B /\left(\pi h_{0}\right)$. The baroclinic momentum is scaled as $\tilde{\mathbf{u}}=\left(T_{0} h_{0}^{2} /\left|f_{0}\right|\right) \tilde{\mathbf{u}}^{*}$ and the vertically integrated velocity $\mathbf{U}$ is scaled by $\pi \Psi_{0} / B=T_{0} /\left|f_{0}\right|$. The dimensional scale of the transport for the case of a flat bottom is of order $T_{0} B^{3} / A_{h} \sim 10^{3} \mathrm{~Sv}$ corresponding to 2200 in dimensionless units. The asterisks are omitted in the main text of the paper. The dimensionless parameters of the model are summarized in Table 1.

\section{B Free waves}

The linearized low-order system describes geostrophic waves in a stratified environment in the presence of topography. Note that $b$ can be regarded as a scaled zonal wavenumber. 
Some prototypes of wave branches are summarized below.

barotropic flat bottom: One finds $U_{0}=0$ and

$$
-\omega^{2} \mathcal{L}^{2} \psi_{C}+\beta^{2} \psi_{C}=0 \quad \rightarrow \quad \omega= \pm \frac{\beta}{\ell^{2}+b^{2}}
$$

for a wave disturbance $\sim \exp i(\ell y-\omega t)$. Then $\pm i \psi_{S}=\psi_{C}$. These are barotropic Rossby waves. With boundary conditions included we get discrete $\ell$.

baroclinic flat bottom: Here, $\phi_{0}=0$ and

$$
-\omega^{2} \phi_{C}+c^{2} \phi_{C}=0 \quad \rightarrow \quad \omega= \pm c= \pm \frac{1}{2} \beta \lambda^{2}
$$

Then $\mp i \phi_{S}=\phi_{C}$ with arbitrary $y$-dependence. These are baroclinic Rossby waves.

barotropic topographic: We find the eigenvalue problem

$$
\omega^{2} \mathcal{L}^{2} \psi_{C}-\beta^{2} \psi_{C}+\frac{1}{2} \mathcal{L} \eta^{2} \psi_{C}=0
$$

Very roughly,

$$
\omega \sim \pm \frac{\beta}{\ell^{2}+b^{2}} \sqrt{1+\frac{1}{2}(\delta / \beta)^{2}}
$$

baroclinic topographic: Consider a purely baroclinic case where the $\psi \equiv 0$. Put $f=-1$ and find

$$
\left[\omega^{2}-c^{2}\right] \phi_{C}+\frac{1}{2} \lambda^{4} \eta\left(\eta \phi_{C}\right)^{\prime \prime}=0
$$

Very roughly,

$$
\omega \sim \pm c \sqrt{1+2(\delta \ell / \beta)^{2}}
$$

mixed barotropic/baroclinic topographic: It is quite complicated to write a single differential equation for the general case of mixed barotropic/baroclinic topographic waves. The forced steady problem is the resistance equation (36). 


\section{Energetics of the low-order system}

The barotropic kinetic energy $U^{2} /(2 h)$ becomes an infinite series in $\eta$ if expressed in the low-order variables. Up to terms $\sim \eta$ we find

$$
\mathcal{E}_{\text {kin }}=\frac{1}{2}\left[U_{0}^{2}+\frac{1}{4}\left(\psi_{C}^{\prime 2}+b^{2} \psi_{C}^{2}\right)+\frac{1}{4}\left(\psi_{S}^{\prime 2}+b^{2} \psi_{S}^{2}\right)\right]+\frac{1}{2} \eta U_{0} \psi_{S}^{\prime}
$$

and the balance of it is found from (9) to (14),

$$
\begin{array}{r}
\frac{\partial \mathcal{E}_{k i n}}{\partial t}=\tau\left(U_{0}+\frac{1}{2} \eta \psi_{S}^{\prime}\right)-\frac{1}{2} \eta U_{0} \phi_{C}-\frac{1}{2} \eta \psi_{C}\left(\phi_{0}+\eta \phi_{S}\right)^{\prime}+ \\
-\mathcal{F}_{k i n}^{\prime}-\frac{1}{2} \epsilon\left[\left(U_{0}^{\prime}\right)^{2}+\left(\mathcal{L} \psi_{C}\right)^{2}+\left(\mathcal{L} \psi_{S}\right)^{2}\right]
\end{array}
$$

It reveals the wind source and dissipative sink and the term describing exchange with the baroclinic energy $\mathcal{E}_{p o t}=\phi_{0}+\eta \phi_{S}$. The latter is derived from the $\mathcal{A}_{0}$-advection term (39). The potential energy balance is (20), or

$$
\frac{\partial \mathcal{E}_{p o t}}{\partial t}=\mathcal{Q}-\mathcal{D}+\frac{1}{2} \gamma \eta \phi_{C} U_{0}+\frac{1}{2} \gamma \eta \psi_{C}\left(\phi_{0}+\eta \phi_{S}\right)^{\prime}-\mathcal{F}_{p o t}^{\prime}
$$

The meridional fluxes $\mathcal{F}_{k i n}$ and $\mathcal{F}_{\text {pot }}$ appearing in these energy balances can be found from (9) to (14) and (20) to (22). The exchange between kinetic and potential energies is thus established by flow over the topography, thereby lifting the stratified water column. It is evident that the advection of potential energy must be retained to achieve a closed balance for total energy $\gamma \mathcal{E}_{k i n}+\mathcal{E}_{\text {pot }}$.

Inspection of the governing equations, either in the full or the simplified form, reveals a second conservation theorem in which the advection terms in baroclinic equations are of third order $\mathrm{n} \delta$ and may be abandoned. It is appropriate for the linearized wave system of the BARBI physics with the above quadratic energy functional $\mathcal{E}_{k i n}$ and

$$
\mathcal{E}_{\text {pot }}^{*}=\frac{1}{2 \lambda^{2}}\left[\left(\phi_{0}+\eta \phi_{S}\right)^{2}+\frac{1}{2} \phi_{C}^{2}+\frac{1}{2} \phi_{S}^{2}\right]
$$

Its rate of change is balanced by external sources, and fluxes $\mathcal{F}_{\text {pot }}^{*}$

$$
\frac{\partial \mathcal{E}_{\text {pot }}^{*}}{\partial t}=(\mathcal{Q}-\mathcal{D})\left(\phi_{0}+\eta \phi_{S}\right)-\frac{1}{2}\left(\eta \psi_{C}\right)^{\prime}\left(\phi_{0}+\eta \phi_{S}\right)+\frac{1}{2} \eta U_{0} \phi_{C}-\left(\mathcal{F}_{p o t}^{*}\right)^{\prime}
$$

